\title{
Spin effects in the effective field theory approach to Post-Minkowskian conservative dynamics
}

\author{
Zhengwen Liu, Rafael A. Porto and Zixin Yang \\ Deutsches Elektronen-Synchrotron DESY, \\ Notkestrasse 85, 22607 Hamburg, Germany \\ E-mail: zhengwen.liu@desy.de, rafael.porto@desy.de, zixin.yang@desy.de
}

ABSTRACT: Building upon the worldline effective field theory (EFT) formalism for spinning bodies developed for the Post-Newtonian regime, we generalize the EFT approach to Post-Minkowskian (PM) dynamics to include rotational degrees of freedom in a manifestly covariant framework. We introduce a systematic procedure to compute the total change in momentum and spin in the gravitational scattering of compact objects. For the special case of spins aligned with the orbital angular momentum, we show how to construct the radial action for elliptic-like orbits using the Boundary-to-Bound correspondence. As a paradigmatic example, we solve the scattering problem to next-to-leading PM order with linear and bilinear spin effects and arbitrary initial conditions, incorporating for the first time finite-size corrections. We obtain the aligned-spin radial action from the resulting scattering data, and derive the periastron advance and binding energy for circular orbits. We also provide the (square of the) center-of-mass momentum to $\mathcal{O}\left(G^{2}\right)$, which may be used to reconstruct a Hamiltonian. Our results are in perfect agreement with the existent literature, while at the same time extend the knowledge of the PM dynamics of compact binaries at quadratic order in spins.

KEywords: Classical Theories of Gravity, Effective Field Theories

ArXiv EPrint: 2102.10059 


\section{Contents}

1 Introduction 1

2 Spinning bodies in the PM EFT approach 4

2.1 Worldline effective theory 4

2.2 Momentum \& spin impulses $\quad 6$

3 Aligned-spin Boundary-to-Bound correspondence $\quad 8$

$\begin{array}{lll}3.1 & \text { Bound radial action I: angle } & 8\end{array}$

$\begin{array}{llr}3.2 & \text { Bound radial action II: impetus } & 9\end{array}$

$\begin{array}{ll}3.3 & \text { Impetus from angle } \\ \end{array}$

4 Scattering to 2PM: linear and bilinear (generic) spin effects 12

$\begin{array}{lll}4.1 & \text { Momentum impulse } & 13\end{array}$

$\begin{array}{lll}4.1 .1 & \text { Leading order } & 13\end{array}$

$\begin{array}{ll}\text { 4.1.2 Next-to-leading order } & 13\end{array}$

$\begin{array}{lll}4.2 & \text { Spin kick } & 14\end{array}$

$\begin{array}{ll}\text { 4.2.1 Leading order } & 14\end{array}$

$\begin{array}{ll}\text { 4.2.2 Next-to-leading order } & 14\end{array}$

$\begin{array}{lll}4.3 & \text { Canonical variables } & 15\end{array}$

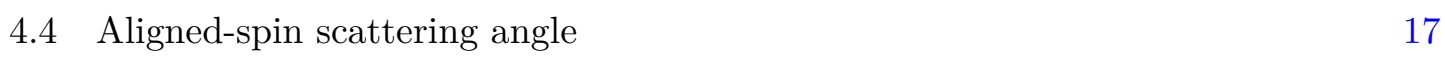

5 Bound states to 2PM: linear and bilinear (aligned) spin effects $\quad 18$

$\begin{array}{lll}5.1 \text { Radial action } & 18\end{array}$

$\begin{array}{ll}5.2 \text { Observables } & 19\end{array}$

$\begin{array}{lll}5.2 .1 & \text { Periastron advance } & 19\end{array}$

$\begin{array}{ll}5.2 .2 \text { Binding energy } & 20\end{array}$

$\begin{array}{ll}5.3 \text { Center-of-mass momentum } & 21\end{array}$

6 Discussion \& outlook 22

A Supplemental material $\quad \mathbf{2 5}$

$\begin{array}{lll}\text { A.1 Trajectories } & 25\end{array}$

A.1.1 Velocity \& position 25

$\begin{array}{lll}\text { A.1.2 Spin } & 26\end{array}$

$\begin{array}{lll}\text { A.2 } & \text { Scattering data } & 27\end{array}$

$\begin{array}{ll}\text { B One-loop integration } & 29\end{array}$ 


\section{Introduction}

The power of gravitational wave (GW) science [1] is predicated on the precise reconstruction of the GW signal as a function of the parameters of the sources, notably binary compact objects [2-4]. As one may anticipate, effects due to spin play a key role in the problem, e.g. [5], particularly due to the expectation that binary black holes in the observable universe may be rapidly rotating, e.g. [6]. Spinning black holes have also attracted interest in recent years due their ability to harvest clouds of putative ultralight particles [7], which can be fleshed out either through mass/spin distributions and GW stochastic backgrounds [8-12] or, more promising, the precise reconstruction of the GW signal emitted from binary systems $[13,14]$. Rotating black holes have puzzled relativists for decades, taking almost 50 years after the discovery of Schwarzschild's solution to arrive at the Kerr metric [15]. Not surprisingly, the situation does not improve in the two-body problem. Consequently, incorporating spin effects in the dynamics of binary systems was a daunting task, with seminal steps in the work of [16-24], and (in comparison) until not that long ago spin effects in the perturbative Post-Newtonian (PN) expansion of small-velocity/weak-gravity were restricted to the spin-orbit sector and to next-to-leading order (NLO) [25-28]. ${ }^{1}$ The state of affairs rapidly progressed thereafter, notably after to the development of the effective field theory (EFT) approach in [30, 31] (see [32-34] for reviews), and spin effects in the PN conservative dynamics are currently known to $\mathrm{N}^{2} \mathrm{LO}[27,35-43]$, and partial results at $\mathcal{O}\left(G^{4}\right)[44,45]$, with generic orientations; and to $\mathrm{N}^{3} \mathrm{LO}[46,47]$ with aligned-spin configurations. $^{2}$ This complements our knowledge of spin-independent conservative contributions both from potential and radiation-reaction effects - which are known up to $\mathrm{N}^{5} \mathrm{LO}$ [62-75], with partial results at higher orders computed using various methodologies, e.g. [76-79]. Many of the calculations representing the current state of the art in the PN regime in particular for the conservative sector and spin effects - were obtained using variants of the EFT approach developed in [30,31]. The goal of this paper is thus to repurpose the worldline theory for spinning bodies, originally introduced for the PN expansion, to calculate spin effects in the Post-Minkowskian (PM) regime using the EFT approach and boundary-to-bound (B2B) correspondence developed in [80-84].

In contrast to applying Mathisson-Papapetrou-Dixon (MPD) equations-of-motion (EoM) independently for momentum and spin [16-19], one of the main advantages of an EFT framework for rotating bodies — presently widely adopted $[34,85]$ — is the introduction of a point-particle effective action to describe compact objects in gravitational backgrounds [31]. ${ }^{3}$ In addition, finite-size effects can be readily incorporated as a series of corrections beyond minimal coupling constrained solely by diffeomorphism invariance [30, 31]

\footnotetext{
${ }^{1}$ Needless to say, numerical simulations for spinning black holes in the strongly coupled regime are also significantly more involved than non-rotating counterparts, e.g. [29].

${ }^{2}$ On the other hand, spin-dependent radiation effects are only known to NLO to quadratic order in the spins [28, 48-55], and $\mathrm{N}^{2} \mathrm{LO}$ for spin-orbit corrections [56]. The radiated power without spin was (re)obtained in the EFT framework of $[30,57]$ to $\mathrm{N}^{2} \mathrm{LO}$ in [58]. Absorption effects in the EFT were studied in [59-61].

${ }^{3}$ The use of a Lagrangian for spinning bodies in general relativity was also advocated in the earlier work of $[21,86]$, see also e.g. [87] and references therein for other developments following an action principle.
} 
without the need of an ansatz for the stress energy tensor. The EFT framework therefore reduces the number of free parameters in previous (more traditional) approaches, e.g. [40]. There are a few other subtleties when dealing with the spin dynamics of point-like objects. For instance, the gauge redundancy in describing rotational degrees of freedom in relativistic theories means that Spin Supplementarity Conditions (SCCs) are often invoked [88]. Moreover, rather than a position the spin angular momentum behaves as a conjugated variable. This naturally leads us to an effective theory written in terms of a Routhian [36-38]; that is, half as a Lagrangian (for the position/velocity) and half as a Hamiltonian (for the spin variables). The EFT machinery then sets in, systematically "integrating out" in the saddle point approximation the potential and radiation modes of the gravitational field, via a series of Feynman diagrams. As it is customary, divergences of the point-particle approximation are then naturally handled via regularization/renormalization [34].

Up until recently, efforts to solve the conservative binary dynamics of compact objects have focused on the direct calculation of the Hamiltonian [68] or Lagrangian [69-73] as an intermedia step towards constructing waveforms. This was no different for spin effects [34, 85]. However, building upon novel ideas from scattering amplitudes [89-91], in the last years we experienced an explosion of work using the classical limit of amplitudes, either to compute the impulse or to extract an effective Hamiltonian which can then be used to study generic orbits, e.g. [92-134]. These developments, which notably belong to the realm of the PM expansion, have produced the state of the art for the conservative dynamics of non-spinning bodies in the PM regime at $\mathrm{N}^{2} \mathrm{LO}$ order [93-95], and very recently also partial results (with potential modes) at $\mathrm{N}^{3} \mathrm{LO}$ [134]. On the other hand, for spinning bodies, spin-orbit and $\operatorname{spin}_{1}$-spin 2 contributions are presently known to NLO in the PM regime $[97,104,105,121,122,135,136]$. (Radiation effects in the PM expansion have also been recently approached in e.g. [137-141].)

While the derivation of a (classical) Hamiltonian from a (quantum) scattering amplitude as an intermedia step is a perfectly viable option (dating back to the work of Iwasaki [142]), one of the main paradigms in modern approaches is to avoid the introduction of gauge-dependent objects [89, 90]. For instance, this was adopted in [96, 97] to solve the (classical) scattering problem, obtaining the impulse from the amplitude, although without providing yet the necessary link to bound states. This became then the main motivation behind the $\mathrm{B} 2 \mathrm{~B}$ correspondence - to remain entirely within the on-shell philosophy. The B2B dictionary was first introduced in [80, 81], mapping scattering data to observables for elliptic-like orbits via a radial action and analytic continuation in binding energy and angular momentum. This allowed us to directly relate gravitational observables without ever invoking a Hamiltonian. In its first incarnation [80], the B2B map relied on the connection (dubbed 'impetus formula') between the center-of-mass momentum and the (infrared-finite) scattering amplitude in the classical limit, which was used to compute the radial action. (See [134] for further developments in the amplitude-action link motivated by the B2B correspondence $[80,81]$.)

In the second version, suggested in [80] and elaborated upon in [81], the B2B map was entirely constructed from the knowledge of the scattering angle instead. Moreover, in [81] we showed how spin effects are incorporated in the B2B correspondence through the connec- 
tion between the periastron advance and scattering angle, albeit for configurations where spins are aligned with the orbital angular momentum. Once the B2B dictionary is written in terms of the deflection angle, bypassing the need to go through the classical limit of a scattering amplitude, the remaining task is to systematically compute the former entirely within the classical domain. Following the pioneering work in [30], an EFT formalism was developed in [82] to solve for the impulse and scattering angle via Feynman diagrams, originally without spin effects. Shortly after the EFT approach was introduced, and benefiting from the simplifications of the classical framework together with powerful tools for computing 'loop' integrals via differential equations [91, 128], the EFT formalism rapidly achieved the state of the art at 3PM [83], subsequently yielding new results for tidal effects beyond leading order [84]. In this paper, building upon the worldline theory in [31, 37, 38, 41], we continue the development of the EFT approach by incorporating spin effects in the scattering problem, insofar for the conservative sector. We will then implement the B2B dictionary to derive observables for bound orbits with aligned spins. Mirroring the simplifications already reflected in [82-84], the inclusion of spin in classical scattering and consequently in elliptic-like motion become remarkably simpler than computing the Hamiltonian. As a result, we will readily achieve the state of the art for spin-orbit and $\operatorname{spin}_{1}$-spin ${ }_{2}$ effects in the PM regime, and present $\operatorname{spin}_{1(2)}-$ Spin $_{1(2)}$ contributions to NLO, including finite-size effects, for the first time.

This paper is organized as follows. In section 2 we review the worldline EFT for spinning compact objects developed in [31, 37, 38, 41], and subsequently adapt it to the PM expansion along the lines of [82]. In section 3 we discuss the construction of the bound radial action via the $\mathrm{B} 2 \mathrm{~B}$ map with (aligned-)spin effects, as well as the matching between the coefficients of the deflection angle and the square of the CoM momentum (impetus). In section 4 we apply the EFT approach within the covariant SSC to compute the total momentum impulse and spin kick to $2 \mathrm{PM}$ and quadratic order in the spins, with generic initial orientations. We also discuss the map to canonical variables and derive the (alignedspin) deflection angle. Finally, in section 5 we derive the bound radial action through the $\mathrm{B} 2 \mathrm{~B}$ correspondence and compute observables to NLO in the PM regime and quadratic order in the spins, including finite-size effects beyond leading order. We also display the coefficients of the impetus to $2 \mathrm{PM}$. We conclude in section 6 with a few remarks on future directions. Aspects of the calculations are relegated to appendices. We provide also an ancillary file as Supplementary Material with more detailed results.

Conventions. We use $\eta_{\mu \nu}=\operatorname{diag}(+,-,-,-)$ for the Minkowski metric. The product of four-vectors is denoted as $k \cdot x=\eta_{\mu \nu} k^{\mu} x^{\nu}$, and $\boldsymbol{k} \cdot \boldsymbol{x}=\delta^{i j} \boldsymbol{k}^{i} \boldsymbol{x}^{j}$ for the Euclidean case, with boldface letters representing three-vectors. We use the convention $\epsilon_{0123}=1$ from the xCoba package. We work in dimensional regularization in $D=d-2 \epsilon$ dimensions, with $d$ either 4,3 or 2 . We use the notation $\int_{k} \equiv \int d^{D} k /(2 \pi)^{D}$, as well as $\hat{\delta}(x) \equiv 2 \pi \delta(x)$. We use $M_{\mathrm{Pl}}^{-1} \equiv \sqrt{32 \pi G}$ for the Planck mass, in $\hbar=c=1$ units, with $G$ Newton's constant. 


\section{Spinning bodies in the PM EFT approach}

We start by briefly reviewing the worldline effective theory approach for rotating compact bodies $[31,37,38]$. Afterwards we show how to solve for the momentum and spin impulse in gravitational scattering to all orders in $G$. For more details in the EFT framework see [34].

\subsection{Worldline effective theory}

As it is well-known, e.g. [88], additional constrained variables are needed in order to introduce a local (off-shell) effective action describing a spinning body in a relativistic framework. Following the analogy with angular momentum, we use a spin tensor, $S^{\alpha \beta}$, that is subject to a SSC (technically a second class constraint) [31]. In order to preserve covariance (without background fields) it is customary to resort to a covariant one,

$$
S^{\alpha \beta} p_{\beta}=0,
$$

with $p_{\mu}$ the particle's momentum. The preservation of the SSC upon evolution implies [34]

$$
p^{\alpha}=\frac{1}{\sqrt{v^{2}}}\left(m v^{\alpha}+\frac{1}{2 m} R_{\beta \rho \mu \nu} S^{\alpha \beta} S^{\mu \nu} v^{\rho}+\cdots\right),
$$

with $v^{\mu} \equiv \frac{d x^{\mu}}{d \sigma}$ the particle's velocity and $\sigma$ as an affine parameter. The ellipses account for higher orders in spin and curvature. To bilinear order in the spin, the SSC in (2.1) becomes

$$
S^{\alpha \beta} v_{\beta}=0+\mathcal{O}\left(S^{3}\right) .
$$

The mass, $m \equiv m\left(S^{2}\right)$, can be read-off from the on-shell condition, $p^{2}=m^{2}$, which also serves as a constraint enforcing reparameterization invariance [88].

To introduce a worldline action, from which the equations of motion can be derived, it is convenient to use a tetrad field, $e_{\mu}^{I}$, which co-rotates with the (compact) body [31]. Using a locally-flat frame, $e_{\mu}^{a}$ (with $g^{\mu \nu} e_{\mu}^{a} e_{\nu}^{b}=\eta^{a b}$ ), the co-rotating tetrad can be parameterized in terms of an element of the Lorentz algebra, $\Lambda_{a}^{I}$, via $e_{\mu}^{I}=\Lambda_{a}^{I} e_{\mu}^{a}$. Using these fields (and time derivatives) as degrees of freedom we can introduce an action such that we obtain the MPD equations of motions, with the spin tensor emerging as a momentum variable conjugate to the angular velocity of the co-rotating field [31]. Because of this, rather than a Lagrangian (or a Hamiltonian), it turns out to be useful to use a Routhian instead to describe rotating bodies, with the spin promoted to a lead-actor in the effective theory. Furthermore, it is also convenient to write the worldline theory using the spin variables projected onto the locally-flat frame,

$$
S^{a b} \equiv S^{\mu \nu} e_{\mu}^{a} e_{\nu}^{b}
$$

such that the $S^{a b}$ matrices obey the $\mathrm{SO}(1,3)$ algebra,

$$
\left\{S^{a b}, S^{c d}\right\}=\eta^{a c} S^{b d}+\eta^{b d} S^{a c}-\eta^{a d} S^{b c}-\eta^{b c} S^{a d} .
$$

The SSC is then easily incorporated through Lagrange multipliers which are fixed by the preservation upon evolution. These extra parameters then yield an additional (curvaturedependent) term in the worldline Routhian (see (2.8) below) [34]. As in the non-spinning 
case, the worldline theory can also readily include spin-dependent finite-size effects through diffeomorphism invariant contributions beyond minimal coupling [30, 31]. For instance, the self-induced quadrupole moment of a rotating body is described by the coupling, first introduced in [31, 37, 38],

$$
\frac{C_{E S^{2}}}{2 m} \int \frac{E_{\mu \nu}}{\sqrt{v^{2}}} e_{a}^{\mu} e_{b}^{\nu} S^{a c} S_{c}^{b} d \sigma
$$

where $E_{\mu \nu}$ is the electric compoment of the Weyl tensor. The Wilson coefficient, $C_{E S^{2}}$, parameterizes our ignorance about the internal degrees of freedom of the compact object, either a black hole, neutron star, or any other exotic possibility. For example, for a Kerr black hole we have $C_{E S^{2}}^{\text {Kerr }}=1$ [38], but (much) larger values may be obtained in other scenarios, for instance with clouds of ultralight particles surrounding black holes [13, 14].

Before we move on, there is yet another important simplification that occurs when studying scattering processes. As it was discussed in [82-84], without spin, we can introduce an einbein, $e$, and a Polyakov-type action linear in the metric field. We can then choose the gauge $e=1$, which coincides with the proper-time for incoming and outgoing states. It is straightforward to extend the same reasoning to the case of spinning bodies, resulting in a point-particle wordline action that can be written as

$$
S_{\mathrm{pp}} \equiv \int_{-\infty}^{+\infty} d \tau \mathcal{R}
$$

with $\tau$ the proper-time (at $\pm \infty$ ). The Routhian, $\mathcal{R}$, in the covariant SSC then takes the form

$$
\begin{aligned}
\mathcal{R}=-\frac{1}{2}( & m g_{\mu \nu} v^{\mu} v^{\nu}+\omega_{\mu}^{a b} S_{a b} v^{\mu} \\
& \left.\quad+\frac{1}{m} R_{\beta \rho \mu \nu} e_{a}^{\alpha} e_{b}^{\beta} e_{c}^{\mu} e_{d}^{\nu} S^{a b} S^{c d} v^{\rho} v_{\alpha}-\frac{C_{E S^{2}}}{m} E_{\mu \nu} e_{a}^{\mu} e_{b}^{\nu} S^{a c} S_{c}{ }^{b}+\cdots\right),
\end{aligned}
$$

to linear order in curvature and quadratic order in the spins, with $\omega_{\mu}^{a b}$ the Ricci rotation coefficients. The last two (curvature-dependent) terms account for the conservation of the SSC as well as finite-size effects to quadratic order in the spins, respectively. The EoM are obtained via [34]

$$
\frac{\delta}{\delta x^{\mu}} S_{\mathrm{pp}}=0, \quad \frac{d}{d \tau} S^{a b}=\left\{S^{a b}, \mathcal{R}\right\}
$$

Notice that after expanding in the weak field limit,

$$
g_{\mu \nu}=\eta_{\mu \nu}+h_{\mu \nu} / M_{\mathrm{Pl}},
$$

the mass coupling remains linear in the metric [82], but that is not the case for the other terms, which instead yield non-linear gravitational interactions both at linear and bilinear order in the spin. In what follows we show how to use this formalism to compute the total momentum and spin impulses in gravitational encounters. 


\subsection{Momentum \& spin impulses}

The computation follows similar steps as described in [82]. We start by 'integrating out' the gravitational field in the potential region in a saddle-point approximation $(A=1,2)$

$$
e^{i S_{\mathrm{eff}}\left[x_{A}, S_{A}^{a b}\right]}=\int \mathcal{D} h_{\mu \nu} e^{i S_{\mathrm{EH}}[h]+i S_{\mathrm{GF}}[h]+i \int d \tau \mathcal{R}\left[x_{A}, S_{A}^{a b}, h\right]},
$$

where $S_{\mathrm{EH}}$ and $S_{\mathrm{GF}}$ are the Einstein-Hilbert action and gauge-fixing terms, respectively. As explained in [82], we adapt $S_{\mathrm{GF}}$ (as well as total time-derivatives) to simplify the resulting Feynman rules. The effective Routhian/action then becomes a (local-in-time) ${ }^{4}$ function of the position and spin of the two-body systems,

$$
S_{\text {eff }}=\sum_{n} \int d \tau_{1} \mathcal{R}_{n}\left[x_{1}\left(\tau_{1}\right), S_{1}\left(\tau_{1}\right) ; x_{1}\left(\tau_{2}\right), S_{2}\left(\tau_{1}\right)\right]
$$

The $\mathcal{R}_{n}$ 's are the $\mathcal{O}\left(G^{n}\right)$ contribution to the worldline Routhian after evaluating the Feynman integrals for generic configurations. From here we can then use (2.9) to obtain the EoM, which we can solve iteratively in powers of $G$, both for the position variables [82],

$$
\begin{aligned}
& x_{A}^{\mu}\left(\tau_{A}\right)=b_{A}^{\mu}+u_{a}^{\mu} \tau_{a}+\sum_{n} \delta^{(n)} x_{A}^{\mu}\left(\tau_{A}\right), \\
& v_{A}^{\nu}\left(\tau_{A}\right)=u_{A}^{\nu}+\sum_{n} \delta^{(n)} v_{A}^{\nu}\left(\tau_{A}\right),
\end{aligned}
$$

as well as the spin in a locally-flat frame,

$$
S_{A}^{a b}\left(\tau_{A}\right)=\mathcal{S}_{A}^{a b}+\sum_{n} \delta^{(n)} S_{A}^{a b}\left(\tau_{A}\right) .
$$

The initial values, $\left\{b_{A}^{\mu}, u_{A}^{\mu}, \mathcal{S}_{A}^{a b}\right\}$, are related to the impact parameter, $b \equiv b_{1}-b_{2}$, incoming velocity and spin, respectively. Since the perturbation vanishes at infinity, we have $e_{\mu}^{a} \rightarrow \delta_{\mu}^{a}$. Hence, the locally-flat frame and Lorentzian one (where the initial spins and velocities are defined) coincide. This observation allows us to enforce the SSC in (2.3) via the constraint

$$
\mathcal{S}_{\mu \nu} u^{\nu}=0
$$

on the initial data, which is then preserved by the evolution equations in (2.9). The total momentum impulse is obtained as in [82], but with a Routhian rather than a Lagrangian,

$$
\Delta p_{A}^{\mu}=-\eta^{\mu \nu} \sum_{n} \int_{-\infty}^{+\infty} \mathrm{d} \tau_{A} \frac{\partial \mathcal{R}_{n}}{\partial x_{A}^{\nu}}
$$

Similarly to the non-spinning case, the iterations of the EoM on lower order contributions to the effective action play an important role [82], and we have the same type of decomposition

$$
\Delta^{(n)} p_{A}^{\mu}=\sum_{k \leq n} \Delta_{\mathcal{R}_{k}}^{(n)} p_{A}^{\mu}
$$

\footnotetext{
${ }^{4}$ We are ignoring here the non-local contributions due to radiation-reaction (tail) effects [65, 67, 71], which include also spin-dependent effects at higher PM orders.
} 
at $n \mathrm{PM}$ order, with

$$
\begin{aligned}
\Delta_{\mathcal{R}_{k}}^{(n)} p_{A}^{\mu} \equiv & -\eta^{\mu \nu} \int_{-\infty}^{+\infty} \mathrm{d} \tau_{A} \\
& \times\left(\frac{\partial}{\partial x_{A}^{\nu}} \mathcal{R}_{k}\left[b_{A(B)}+u_{A(B)} \tau_{A(B)}+\sum_{r=0}^{n-k} \delta^{(r)} x_{A(B)} ; \mathcal{S}_{A(B)}^{a b}+\sum_{r=0}^{n-k} \delta^{(r)} S_{A(B)}^{a b}\right]\right)_{\mathcal{O}\left(G^{n}\right)}
\end{aligned}
$$

Likewise, the total change of spin follows from

$$
\Delta S_{A}^{a b}=\sum_{n} \int_{-\infty}^{+\infty} d \tau_{A}\left\{S_{A}^{a b}, \mathcal{R}_{n}\right\}
$$

which must be evaluated iteratively on solutions to the EoM, yielding the same structure, i.e. $\Delta_{\mathcal{R}_{k}}^{(n)} S_{A}^{a b}$, as in $(2.18)$.

It is somewhat convenient to re-write the final covariant expressions, obtained after using the EoM though (2.9), in terms of the initial Pauli-Lubanski vector,

$$
\mathcal{S}_{A}^{\mu}=m_{A} a_{A}^{\mu} \equiv \frac{1}{2} \epsilon^{\mu}{ }_{\nu \alpha \beta} \mathcal{S}_{A}^{\alpha \beta} u_{A}^{\nu},
$$

where we take advantage of the fact that both the incoming velocity and spin tensor live in the same (inertial) frame. This observation drastically simplifies the handling of the SSC in the scattering problem. By considering only incoming/outgoing states in Minkowski space, the complexity due to the mismatch between the locally-flat and 'PN frame' disappears. Furthermore, for the case of spins aligned with the angular momentum, it is easy to see that the motion remains in a plane, and we can compute the standard deflection angle, e.g. [82],

$$
2 \sin \left(\frac{\chi}{2}\right)=\frac{\sqrt{-\Delta p_{1}^{2}}}{p_{\infty}},
$$

where the momentum at infinity, $p_{\infty}$, is given by

$$
p_{\infty}=\mu \frac{\sqrt{\gamma^{2}-1}}{\Gamma}=\mu \hat{p}_{\infty}
$$

and

$$
\begin{aligned}
\gamma & \equiv u_{1} \cdot u_{2}, \\
\Gamma & \equiv E / M=\sqrt{1+2 \nu(\gamma-1)},
\end{aligned}
$$

with $E$ the total energy in the CoM frame. Throughout the remaining of this paper we use the notation $M=m_{1}+m_{2}$ for the total mass, $\mu=m_{1} m_{2} / M$ for the reduced mass, and $\nu \equiv \mu / M$ for the symmetric mass ratio. We also introduce the (reduced) binding energy, $\mathcal{E}$, such that

$$
E=M(1+\nu \mathcal{E}) .
$$




\section{Aligned-spin Boundary-to-Bound correspondence}

In principle, the B2B dictionary with generic spins would require a map for non-planar motion. However, a major simplification arises for aligned-spin configurations, which we have shown in [81] is amenable to the same correspondence between the periastron advanced, $\Delta \Phi$, and scattering angle, $\chi$,

$$
\frac{\Delta \Phi(J, \mathcal{E})}{2 \pi}=\frac{\chi(J, \mathcal{E})+\chi(-J, \mathcal{E})}{2 \pi}, \quad \mathcal{E}<0,
$$

albeit with the canonical total angular momentum, $\boldsymbol{J} \equiv \boldsymbol{L}+\boldsymbol{S}_{1}+\boldsymbol{S}_{2}$, as opposite to the orbital angular momentum, $\boldsymbol{L}$, which enters in the non-spinning case. We review in what follows how to use (3.1) to reconstruct the bound radial action from scattering data for aligned spins. For convenience, we will write various results in terms of the spin vector in (2.20), which for aligned spins obeys $a_{A}^{\mu} u_{A \mu}=a_{A}^{\mu} b_{\mu}=0$, and introduce the scalar variables $a_{A} \equiv \boldsymbol{a}_{A} \cdot \boldsymbol{L}$. Moreover, we often use the spin parameters $a_{ \pm}=a_{1} \pm a_{2}$ for the two-body state, as well as the re-scaled variables $\tilde{a}_{ \pm} \equiv a_{ \pm} /(G M), \ell \equiv L / G M \mu$ for the spin and orbital angular momentum.

\subsection{Bound radial action I: angle}

As it was shown in [81], for the case of non-spinning bodies the relationship in (3.1) allows us to construct the (reduced) radial action for the bound problem, $i_{r}(\mathcal{E}, \ell)$, in terms of the analytic continuation to negative binding energy of the PM coefficient of the scattering angle,

$$
\frac{\chi}{2}=\sum_{n} \chi_{b}^{(n)}(\mathcal{E})\left(\frac{G M}{b}\right)^{n}=\sum_{n} \frac{\chi_{\ell}^{(n)}(\mathcal{E})}{\ell^{n}},
$$

yielding $\left(\right.$ with $\left.\operatorname{sg}\left(\hat{p}_{\infty}\right) \equiv \hat{p}_{\infty} / \sqrt{-\hat{p}_{\infty}^{2}}\right)$

$$
i_{r}(\mathcal{E}, \ell)=\operatorname{sg}\left(\hat{p}_{\infty}\right) \chi_{\ell}^{(1)}(\mathcal{E})-\ell\left(1+\frac{2}{\pi} \sum_{n=1} \frac{\chi_{\ell}^{(2 n)}(\mathcal{E})}{(1-2 n) \ell^{2 n}}\right) \quad \text { (without spin) } .
$$

The expression in (3.3) does not translate directly to the spinning case. For starters, the expansion in (3.2) gets modified into a two-scale expansion with spin effects, such that in addition to the standard factors of $G M / b$ we also have an expansion in $a_{ \pm} / b$. This can be circumvented by the introduction of the dimensionless variables $\tilde{a}_{ \pm}=a_{ \pm} /(G M)$, which allows us to conveniently keep the same type of expansion as in $(3.2)$ (with $\chi_{\ell}^{(n)}\left(\mathcal{E}, \tilde{a}_{ \pm}\right.$) coefficients) at the expenses of a minor mismatch in the $G$ power-counting. Hence, using the fact that the relationship in (3.1) involving both the orbital and spin angular momentum still applies, we can once again integrate with respect to $L$ and perform the same manipulations as in [81] to obtain, after some trivial re-arrangement,

$$
i_{r}\left(\mathcal{E}, \ell, \tilde{a}_{ \pm}\right)=\operatorname{sg}\left(\hat{p}_{\infty}\right) \chi_{\ell}^{(1)}(\mathcal{E})+\ell\left(-1+\frac{2}{\pi} \sum_{n=1}^{\infty}\left(\frac{\chi_{\ell, \text { odd }}^{(2 n+1)}\left(\mathcal{E}, \tilde{a}_{ \pm}\right)}{2 n \ell^{2 n+1}}+\frac{\chi_{\ell, \text { even }}^{(2 n)}\left(\mathcal{E}, \tilde{a}_{ \pm}\right)}{(2 n-1) \ell^{2 n}}\right)\right)
$$

The $\chi_{\ell, \text { odd (even) }}^{(k)}\left(\mathcal{E}, \tilde{a}_{ \pm}\right)$are the odd (and even) contributions in the $\tilde{a}_{ \pm}$spin variables, analytically continued to negative binding energies. The expression in (3.4) plays a similar role 
as (3.3), with the addition of the odd contributions accounting for spin-orbit corrections. The even terms including not only spin-independent factors, but also effects quadratic in spin. Higher orders in spin follow the same pattern.

There is still an important caveat in the B2B dictionary for spinning bodies. The solution to the scattering problem produces results in an expansion in $G M / b$, with $b$ the covariant impact parameter, as in (3.2). However, for rotating bodies the latter is not directly related to $\ell$, the canonical orbital angular momentum. Instead we have $[135,136]$

$$
\ell=\hat{p}_{\infty} \frac{b}{G M}+\frac{\Gamma-1}{2 \nu}\left(\tilde{a}_{+}-\frac{\delta}{\Gamma} \tilde{a}_{-}\right)
$$

where $\delta \equiv \sqrt{1-4 \nu}\left(m_{1}-m_{2}\right) /\left|m_{1}-m_{2}\right|$. This introduces an additional expansion in $\tilde{a}_{ \pm} / \ell$ once the scattering angle in (3.2) is written in covariant form, mixing the power-counting. For example, it leads to spin-dependent contributions stemming off of the spin-independent deflection angle in impact-parameter space [81].

\subsection{Bound radial action II: impetus}

As it was demonstrated in $[80,81]$, the B2B dictionary relies on the connection between the orbital elements for hyperbolic- and elliptic-like motion [80, 81]. The orbital elements are obtained from the roots of the radial momentum, which can be solved as a function of the binding energy using a gauge where the (canonical) impetus takes the quasi-isotropic form

$$
P_{r}^{2}=p_{\infty}^{2}\left(1+\sum_{i=1}^{\infty} f_{i}\left(\mathcal{E}, \ell \tilde{a}_{ \pm}, \tilde{a}_{ \pm}^{2}, \cdots\right) \frac{(G M)^{i}}{r^{i}}\right)-\frac{L^{2}}{r^{2}}
$$

The expression in (3.6) also allows us to re-write the radial action in terms of the $f_{i}$ 's, using the same algebraic relationships uncovered in [80, 81]. For instance, for the case of non-spinning bodies, the coefficients in the PM expansion of (3.2) are related to the CoM momentum in (3.6), via [80]

$$
\chi_{\ell}^{(n)}(\mathcal{E})=\frac{\sqrt{\pi}}{2} \hat{\Gamma}\left(\frac{n+1}{2}\right) \sum_{\sigma \in \mathcal{P}(n)} \frac{\hat{p}_{\infty}^{n}}{\Gamma\left(1+\frac{n}{2}-\Sigma^{k}\right)} \prod_{k} \frac{f_{\sigma_{k}}^{\sigma^{k}}(\mathcal{E})}{\sigma^{k} !} \quad \text { (without spin) }
$$

which follows from Firsov's solution to the scattering problem [143]. (See [80] for details on the combinatorial manipulations involved in (3.7).) Using the expression in (3.3), the relation in (3.7) then leads to an alternative representation for the radial action - so far for the case of non-spinning bodies. However, as demonstrated in [81] (see its appendix A), the resulting form in terms of the $f_{i}$ 's coincides with the PM expansion of the radial action that follows from the direct integration of the radial momentum, i.e.

$$
i_{r}\left(\ell, \mathcal{E}, \tilde{a}_{ \pm}\right)=\frac{1}{2 \pi G M \mu} \oint P_{r}\left(\ell, \mathcal{E}, \tilde{a}_{ \pm}\right) d r, \quad \text { (bound) }
$$

using Sommerfeld's contour in the complex plane (originally performed to all orders in [80]). Hence, after noticing that the spin and angular momentum are simple spectators in all manipulations involving integration over the radial coordinate, it is straightforward to 
conclude that the general solution for the radial action in terms of the coefficients of the CoM momentum (in isotropic gauge) carries over unscathed onto the spinning case, obtaining

$$
\begin{aligned}
& i_{r}\left(\mathcal{E}, \ell, \tilde{a}_{ \pm}\right)=\frac{\hat{p}_{\infty}^{2}}{\sqrt{-\hat{p}_{\infty}^{2}}} \frac{f_{1}}{2}+ \frac{\ell}{2 \sqrt{\pi}} \sum_{n=0}^{\infty}\left(\frac{\hat{p}_{\infty}}{\ell}\right)^{2 n} \Gamma\left(\frac{2 n-1}{2}\right) \quad \text { (with spin) } \\
& \times \sum_{\sigma \in \mathcal{P}(2 n)} \frac{1}{\Gamma\left(1+n-\Sigma^{k}\right)} \prod_{k} \frac{f_{\sigma_{k}}^{\sigma^{k}}\left(\mathcal{E}, \ell, \tilde{a}_{ \pm}\right)}{\sigma^{k !}}
\end{aligned}
$$

in terms of the coefficients in (3.6). For instance, we have [80, 81]

$$
\begin{aligned}
i_{r}\left(\mathcal{E}, \ell, \tilde{a}_{ \pm}\right)=-\ell & +\frac{\hat{p}_{\infty}^{2}}{\sqrt{-\hat{p}_{\infty}^{2}}} \frac{f_{1}}{2}+\frac{\hat{p}_{\infty}^{2}}{2 \ell} f_{2}+\frac{\hat{p}_{\infty}^{4}}{4 \ell^{3}}\left(\frac{f_{2}^{2}}{2}+f_{1} f_{3}+f_{4}\right) \\
& +\frac{\hat{p}_{\infty}^{6}}{16 \ell^{5}}\left(f_{2}^{3}+6\left(f_{1} f_{3}+f_{4}\right) f_{2}+3\left(f_{4} f_{1}^{2}+2 f_{5} f_{1}+f_{3}^{2}+f_{6}\right)\right) \\
& +\frac{5 \hat{p}_{\infty}^{8}}{128 \ell^{7}}\left(\cdots+6 f_{1}^{2} f_{3}^{2}+\cdots\right)+\cdots,
\end{aligned}
$$

where we kept only the one piece in the final term which will be needed later on. (The reader should keep in mind that the $f_{i}$ 's themselves may also depend on $\ell$.)

Notice that, similarly to the non-spinning case, the $f_{k}$ 's contribute also at $n \mathrm{PM}$ order (for $n \geq k$ ). This will allow us to perform a consistent PN-truncation, as discussed in [8084]. We will return to this point in section 5 .

\subsection{Impetus from angle}

It is useful to relate the PM coefficients in (3.6) to the deflection angle in (3.2), also with spin effects. This will allow us to perform the analytic continuation of the CoM momentum to negative binding energies, and also find a Hamiltonian if so desired. The main observation is the same we used to arrive at the equivalent representation for the radial action in (3.9). That is, spin and angular momentum are going for the ride when the radial action is constructed via the integral of the radial momentum, regardless of whether we consider bound or unbound orbits. Hence, the result of the integral

$$
i_{r}\left(\mathcal{E}, \ell, \tilde{a}_{ \pm}\right)=\frac{1}{2 \pi G M \mu} \int_{-\infty}^{\infty} P_{r}\left(\mathcal{E}, \ell, \tilde{a}_{ \pm}\right) d r \quad \text { (unbound) }
$$

remains also the same, with the $f_{i}$ 's in (3.6) including spin-dependent parts. Moreover, since the scattering angle obeys

$$
-\frac{\partial}{\partial \ell} i_{r}\left(\mathcal{E}, \tilde{a}_{ \pm}\right)=\frac{1}{2}+\frac{\chi\left(\ell, \mathcal{E}, \tilde{a}_{ \pm}\right)}{2 \pi} \quad \text { (unbound) }
$$

we can solve for the unbound radial action, which can then be written as

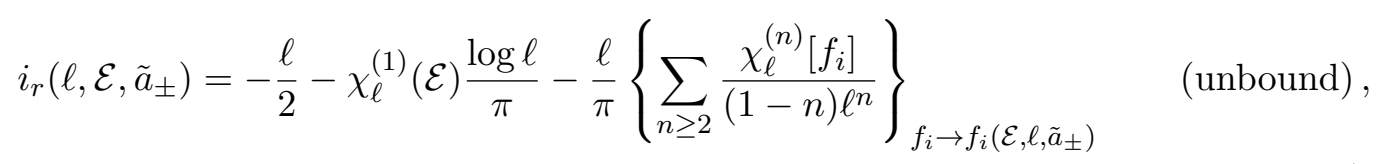

with the functional form of $\chi_{\ell}^{(n)}\left[f_{i}\right]$ given exactly by the expression in (3.7), to all PM orders. 
Let us stress two related important points regarding (3.13). First of all, there could be a constant of integration (depending only on the binding energy) as in the bound case [80]. Moreover, the $n=1$ term $(\propto \log \ell)$ is a bit subtle when considering the analytic continuation. The constant of integration may be fixed by using the expression in (3.9) and imposing

$$
i_{r}^{\text {(bound) }}\left(\mathcal{E}<0, \ell, \tilde{a}_{ \pm}\right)=i_{r}^{\text {(unbound) }}\left(\mathcal{E}<0, \ell, \tilde{a}_{ \pm}\right)-i_{r}^{\text {(unbound) }}\left(\mathcal{E}<0,-\ell,-\tilde{a}_{ \pm}\right),
$$

which follows directly from the B2B relation in (3.1). ${ }^{5}$ However, this requires a choice for the branch of the logarithm, when performing the analytical continuation to negative orbital angular momentum. We find the choice $\log (\ell) / \pi-\log (-\ell) / \pi \rightarrow \mp i$, in combination with $\hat{p}_{\infty} \rightarrow \pm i \hat{p}_{\infty}$ for the analytic continuation in the binding energy, leads to

$$
-\chi_{\ell}^{(1)}(\mathcal{E})\left(\frac{\log \ell}{\pi}-\frac{\log (-\ell)}{\pi}\right) \rightarrow \operatorname{sg}\left(\hat{p}_{\infty}\right) \chi_{\ell}^{(1)}(\mathcal{E})
$$

uniquely fixing the unbound radial action. ${ }^{6}$

For convenience, since we work here to quadratic order in the spins, in what follows we decompose the coefficients of the CoM momentum as

$$
f_{i}\left(\mathcal{E}, \ell, \tilde{a}_{ \pm}\right)=f_{i}^{0}(\mathcal{E})+\ell \sum_{A= \pm} \tilde{a}_{A} f_{i}^{A}(\mathcal{E})+\sum_{\{A, B\}= \pm} \tilde{a}_{A} \tilde{a}_{B} f_{i}^{A B}(\mathcal{E})+\cdots
$$

with $f_{i}^{0}(\mathcal{E})$ the spin-independent part, and $\left\{f_{i}^{A}(\mathcal{E}), f_{i}^{A B}(\mathcal{E})\right\}$ (dimensionless) functions of the binding energy (and masses). Likewise for the coefficients in (3.2),

$$
\chi_{\ell}^{(n)}\left(\mathcal{E}, \tilde{a}_{ \pm}\right)=\chi_{0}^{(n)}(\mathcal{E})+\sum_{A= \pm} \tilde{a}_{A} \chi_{A}^{(n)}(\mathcal{E})+\sum_{\{A, B\}= \pm} \tilde{a}_{A} \tilde{a}_{B} \chi_{A B}^{(n)}(\mathcal{E})+\cdots
$$

(we suppress the $\ell$-subscript on the r.h.s. for notational convenience). Hence, applying (3.12) to (3.13), while keeping track of all the $\ell$ 's inside the $f_{i}$ 's in (3.16), we can derive the scattering angle in terms of the CoM momentum including spin effects to all PM orders. As we will see momentarily, terms linear and quadratic in the spin first show up at $n=2$ and $n=3$ in (3.17), respectively. This is intuitively simple to understand, and it follows directly from the expansion in impact-parameter of the deflection angle yielding extra factors of $a_{ \pm} / b$ once spin is included. ${ }^{7}$ As a consequence,

$$
f_{1,2}^{A}(\mathcal{E})=f_{1,2}^{A B}(\mathcal{E})=0
$$

\footnotetext{
${ }^{5}$ This analytic continuation is behind the relationship between the total radiated energy for unbound orbits and the energy emitted over a period, discussed in [144]. This relationship follows immediately from the B2B map applied to the (local part of the) conservative tail effect [65, 74, 79].

${ }^{6}$ The choice must be uniformly adopted to be consistent with the analytic continuation in the (covariant) impact parameter, which was used in [81] to connect the orbital elements.

${ }^{7}$ Notice the leading linear and quadratic terms, scaling as $(G M / b)\left(a_{ \pm} / b\right)$ and $(G M / b)\left(a_{ \pm} / b\right)^{2}$, have the wrong parity through the B2B map and therefore do not contribute to (3.4).
} 
For the remaining coefficients, we find

$$
\begin{aligned}
\chi_{A}^{(2)} & =\frac{\hat{p}_{\infty}^{3}}{2} f_{3}^{A}, \\
\chi_{A}^{(3)} & =\frac{\pi \hat{p}_{\infty}^{4}}{4}\left(f_{1}^{0} f_{3}^{A}+f_{4}^{A}\right), \\
\chi_{A B}^{(3)} & =\hat{p}_{\infty}^{3} f_{3}^{(A B)} \\
\chi_{A B}^{(4)} & =\frac{3 \pi \hat{p}_{\infty}^{4}}{8}\left(f_{1}^{(0)} f_{3}^{A B}+f_{4}^{A B}+\frac{3 \hat{p}_{\infty}^{2}}{4} f_{3}^{A} f_{3}^{B}\right),
\end{aligned}
$$

where (recall $\tilde{a}_{ \pm}=a_{ \pm} /(G M)$ ) we kept only terms which contribute to $\mathcal{O}\left(G^{2}\right)$. Incidentally, notice these values are consistent with the equivalence between the two representations of the radial action, in (3.4) and (3.9)-(3.10). It is straightforward to invert these equations to obtain the value of the CoM impetus. See section 5 for more details.

\section{Scattering to 2PM: linear and bilinear (generic) spin effects}

In this section we apply the EFT formalism for spinning bodies to compute the total momentum and spin impulses to $2 \mathrm{PM}$ and quadratic order in the spins. The needed topologies are shown in figure 1 to $2 \mathrm{PM}$ order. The vertices at the worldline may include mass and spin couplings, both linear (from the Ricci-rotation coefficients) and bilinear (from the SSC and finite-size terms) in the spins. Because the coupling to the mass is linear in the metric perturbation in our (Polyakov-type) gauge [82], the diagram in figure $1 \mathrm{~b}$ only contributes spin-dependent effects. The 'tree-level' diagram in figure 1a contributes both at $1 \mathrm{PM}$ and $2 \mathrm{PM}$ order, the latter through the iteration of the EoM described in section 2.2. Notice we need both spin-dependent iterations on the spin-independent tree-level and vice versa. The 'one-loop' diagrams shown in figure $1 \mathrm{~b}$ and $1 \mathrm{c}$ is evaluated on the unperturbed solutions. For the sake of comparison, in this section we quote the variation of the spin four-vector, defined as

$$
S_{A}^{\mu} \equiv \frac{1}{2 m_{A}} \epsilon^{\mu}{ }_{\nu \alpha \beta} S_{A}^{\alpha \beta} p_{A}^{\nu}
$$

which coincides with the value in (2.20) at early times. We obtain the spin impulse in terms of the total change in the spin tensor, using the spin algebra in (2.5) on the Routhian/action, in combination with the momentum impulse. As a non-trivial check, the results below can be shown to be consistent with the preservation of the SSC, $S_{\mu} p^{\mu}=0$, the on-shell condition, $p^{2}=m^{2}$, and the constancy of the magnitude of the spin, $S_{\mu} S^{\mu}=a^{2}$, to $2 \mathrm{PM}$ order.

We illustrate the basic ideas and quote the results in what follows, with supplemental material in appendix A, and a few comments on the integration procedure in appendix B. Throughout this section we use the notation $|b| \equiv \sqrt{-b^{\mu} b_{\mu}}$ and $\hat{b}^{\mu} \equiv b^{\mu} /|b|$. Moreover, we 


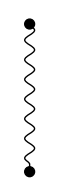

(a)

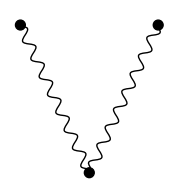

(b)

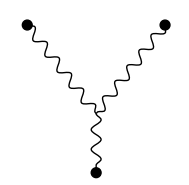

(c)

Figure 1. Feynman topologies needed to 2PM order. (See text.)

also use $\kappa_{ \pm}=C_{E S^{2}}^{(1)} \pm C_{E S^{2}}^{(2)}$, and the tensorial structure [97]

$$
\begin{aligned}
\Pi^{\mu}{ }_{\nu} & \equiv \epsilon^{\mu \rho \alpha \beta} \epsilon_{\nu \rho \gamma \delta} \frac{u_{1 \alpha} u_{2 \beta} u_{1}^{\gamma} u_{2}^{\delta}}{\gamma^{2}-1}, \\
T^{\mu \nu \rho} & \equiv \hat{b}^{\rho} \Pi^{\mu \nu}+\hat{b}^{\nu} \Pi^{\rho \mu}+\hat{b}^{\mu} \Pi^{\rho \nu}, \\
u_{2(1) \perp}^{\mu} & \equiv u_{2(1)}^{\mu}-\gamma u_{1(2)}^{\mu} .
\end{aligned}
$$

\subsection{Momentum impulse}

\subsubsection{Leading order}

The derivation of the tree-level Routhian/action is straightforward. Following the same steps as in [82] and evaluating on the unperturbed solutions in (2.13) and (2.14), we obtain

$$
\Delta_{a_{1}}^{(1)} p_{1}^{\mu}=\frac{\nu G M^{2}}{|b|^{2}} \frac{4 \gamma}{\sqrt{\gamma^{2}-1}} \epsilon_{\alpha \rho \beta \sigma} a_{1}^{\rho} u_{1}^{\beta} u_{2}^{\sigma}\left(\Pi^{\mu \alpha}+2 \hat{b}^{\mu} \hat{b}^{\alpha}\right)-(1 \leftrightarrow 2),
$$

for the spin-orbit contributions, whereas at quadratic order

$$
\Delta_{a_{1} a_{2}}^{(1)} p_{1}^{\mu}=\frac{\nu G M^{2}}{|b|^{3}} \frac{4\left(2 \gamma^{2}-1\right)}{\sqrt{\gamma^{2}-1}} a_{1 \alpha} a_{2 \beta}\left(T^{\alpha \beta \mu}+4 \hat{b}^{\alpha} \hat{b}^{\beta} \hat{b}^{\mu}\right)-(1 \leftrightarrow 2),
$$

and

$$
\Delta_{a_{1}^{2}}^{(1)} p_{1}^{\mu}=\frac{\nu G M^{2}}{|b|^{3}} \frac{2 C_{E S^{2}}^{(1)}\left(2 \gamma^{2}-1\right)}{\sqrt{\gamma^{2}-1}} a_{1 \alpha} a_{1 \beta}\left(T^{\alpha \beta \mu}+4 \hat{b}^{\alpha} \hat{b}^{\beta} \hat{b}^{\mu}\right)-(1 \leftrightarrow 2),
$$

the latter including the insertion of the finite-size term in (2.8). In all of these expressions we have (anti-)symmetrized the result (under which $b^{\mu} \rightarrow-b^{\mu}$ and $\delta \rightarrow-\delta$ ). It is straightforward to show that all of these 1PM values coincide with the results reported in $[97,135,136]$ for the case of Kerr black holes (with $C_{E S^{2}}=1$ ).

\subsubsection{Next-to-leading order}

For the NLO results we must evaluate the one-loop diagrams in figures $1 \mathrm{~b} \& 1 \mathrm{c}$ on the unperturbed solution, and use the trajectories (shown in appendix A) to compute the iteration with the tree-level diagram in figure 1a. The results are:

$$
\begin{aligned}
\Delta_{a}^{(2)} p_{1}^{\mu}= & \frac{\nu G^{2} M^{3}}{|b|^{3}}\left[D_{1} \epsilon_{\alpha \rho \beta \sigma} a_{1}^{\rho} u_{1}^{\beta} u_{2}^{\sigma}\left(\Pi^{\mu \alpha}+3 \hat{b}^{\alpha} \hat{b}^{\mu}\right)\right. \\
& \left.+D_{2} \epsilon^{\mu \alpha \rho \beta} a_{1 \rho} u_{1 \beta} \hat{b}_{\alpha}+\left(a_{1}^{\rho} u_{1}^{\beta} u_{2}^{\sigma} \hat{b}^{\alpha} \epsilon_{\alpha \rho \beta \sigma}\right)\left(D_{3} u_{1}^{\mu}+D_{4} u_{2}^{\mu}\right)\right]-(1 \leftrightarrow 2),
\end{aligned}
$$




$$
\begin{aligned}
\Delta_{a^{2}}^{(2)} p_{1}^{\mu}= & \frac{\nu G^{2} M^{3}}{|b|^{4}}\left[D_{5} a_{1 \alpha} a_{1 \beta}\left(T^{\alpha \beta \mu}+5 \hat{b}^{\alpha} \hat{b}^{\beta} \hat{b}^{\mu}\right)+D_{6} a_{1 \alpha}\left(a_{1} \cdot u_{2}\right)\left(\Pi^{\alpha \mu}+4 \hat{b}^{\alpha} \hat{b}^{\mu}\right)\right. \\
& +a_{1 \alpha} a_{1 \beta}\left(D_{7} u_{1}^{\mu}-D_{8} u_{2}^{\mu}\right)\left(\Pi^{\alpha \beta}+4 \hat{b}^{\alpha} \hat{b}^{\beta}\right)+\hat{b}^{\mu}\left(D_{9} a_{1}^{2}+D_{10}\left(a_{1} \cdot u_{2}\right)^{2}\right) \\
& \left.+2 D_{6} a_{1}^{\mu}\left(a_{1} \cdot u_{2}\right)+\left(a_{1} \cdot u_{2}\right)^{2}\left(D_{11} u_{1}^{\mu}+D_{12} u_{2}^{\mu}\right)-a_{1}^{2}\left(D_{13} u_{1}^{\mu}-D_{14} u_{2}^{\mu}\right)\right] \\
& -(1 \leftrightarrow 2), \\
\Delta_{a_{1} a_{2}}^{(2)} p_{1}^{\mu}= & \frac{\nu G^{2} M^{3}}{|b|^{4}}\left[\frac{1}{2} D_{15} a_{1 \alpha} a_{2 \beta}\left(T^{\alpha \beta \mu}+5 \hat{b}^{\alpha} \hat{b}^{\beta} \hat{b}^{\mu}\right)+D_{16} a_{1 \alpha}\left(a_{2} \cdot u_{1}\right)\left(\Pi^{\alpha \mu}+4 \hat{b}^{\alpha} \hat{b}^{\mu}\right)\right. \\
& +D_{17} a_{1 \alpha} a_{2 \beta} u_{1}^{\mu}\left(\Pi^{\alpha \beta}+4 \hat{b}^{\alpha} \hat{b}^{\beta}\right)+\frac{1}{2} \hat{b}^{\mu}\left(D_{15}\left(a_{1} \cdot a_{2}\right)+D_{18}\left(a_{1} \cdot u_{2}\right)\left(a_{2} \cdot u_{1}\right)\right) \\
& \left.+2 D_{16} a_{1}^{\mu}\left(a_{2} \cdot u_{1}\right)+D_{19}\left(a_{1} \cdot u_{2}\right)\left(a_{2} \cdot u_{1}\right) u_{1}^{\mu}+D_{20}\left(a_{1} \cdot a_{2}\right) u_{1}^{\mu}\right]-(1 \leftrightarrow 2) .
\end{aligned}
$$

The $D_{i}$ coefficients are displayed in appendix A.

\section{$4.2 \quad$ Spin kick}

\subsubsection{Leading order}

We now move to the computation of the spin dynamics. As we discussed earlier, we quote the result in terms of the spin vector in (4.1). We find,

$$
\Delta_{a}^{(1)} S_{1}^{\mu}=-\frac{\nu G M^{2}}{|b|} \frac{2}{\sqrt{\gamma^{2}-1}}\left(\left(\hat{b} \cdot a_{1}\right)\left(u_{1}^{\mu}-2 \gamma u_{2}^{\mu}\right)+2 \gamma \hat{b}^{\mu}\left(a_{1} \cdot u_{2}\right)\right)
$$

at linear order in the spins, while at quadratic order we arrive at

$$
\begin{gathered}
\Delta_{a_{1} a_{2}}^{(1)} S_{1}^{\mu}=\frac{\nu G M^{2}}{|b|^{2}} \frac{2}{\sqrt{\gamma^{2}-1}} \epsilon_{\beta \sigma \rho}^{\mu}\left(\Pi^{\alpha \beta}+2 \hat{b}^{\alpha} \hat{b}^{\beta}\right)\left(u_{1}^{\rho} u_{2}^{\sigma}\left(a_{2 \alpha}\left(a_{1} \cdot u_{2}\right)-\gamma a_{1 \alpha}\left(a_{2} \cdot u_{1}\right)\right)\right. \\
\left.+\gamma^{2} a_{1}^{\rho} a_{2 \alpha} u_{1}^{\sigma}-a_{1 \alpha} a_{2}^{\rho}\left(\left(\gamma^{2}-1\right) u_{1}^{\sigma}+2 \gamma u_{2 \perp}^{\sigma}\right)\right) \\
\Delta_{a^{2}}^{(1)} S_{1}^{\mu}=-\frac{\nu G M^{2}}{|b|^{2}} \frac{2}{\sqrt{\gamma^{2}-1}}\left(\left(2 \gamma^{2}-1\right) C_{E S^{2}}^{(1)} u_{1}^{\rho}+2 \gamma u_{2 \perp}^{\rho}\right) \epsilon_{\beta \sigma \rho}^{\mu} a_{1 \alpha} a_{1}^{\sigma}\left(\Pi^{\alpha \beta}+2 \hat{b}^{\alpha} \hat{b}^{\beta}\right)
\end{gathered}
$$

These results are, once again, in agreement with the 1PM variation obtained in [97] for the case of Kerr black holes.

\subsubsection{Next-to-leading order}

The total change of spin at NLO is significantly more cumbersome. While, based on various arguments, we suspect an underlying structure that extends the compact expressions at 1PM order [135], we have not been able to uncover it so far. Yet, we believe these (manifestly covariant) expressions are perhaps the best hope to unravel a deeper (spacetime) structure. See 6 for more on this point. The results are:

$$
\begin{aligned}
\Delta_{a}^{(2)} S_{1}^{\mu}= & \frac{\nu G^{2} M^{3}}{|b|^{2}}\left[D_{21} a_{1 \alpha}\left(\Pi^{\mu \alpha}+2 \hat{b}^{\alpha} \hat{b}^{\mu}\right)-D_{21} a_{1}^{\mu}+D_{1}\left(\left(\hat{b} \cdot a_{1}\right) u_{2 \perp}^{\mu}-\hat{b}^{\mu}\left(a_{1} \cdot u_{2}\right)\right)\right. \\
& \left.+\left(D_{22} u_{1}^{\mu}+D_{23} \hat{b}^{\mu}\right)\left(\hat{b} \cdot a_{1}\right)+\left(D_{24} u_{1}^{\mu}+D_{25} u_{2 \perp}^{\mu}\right)\left(a_{1} \cdot u_{2}\right)\right]
\end{aligned}
$$




$$
\begin{aligned}
& \Delta_{a_{1} a_{2}}^{(2)} S_{1}^{\mu}=\frac{\nu G^{2} M^{3}}{|b|^{2}} \epsilon^{\mu}{ }_{\nu \alpha \beta}\left[-D_{28} u_{1}^{\nu} u_{2}^{\beta} a_{1 \sigma} a_{2 \rho}\left(T^{\alpha \rho \sigma}+4 \hat{b}^{\alpha} \hat{b}^{\rho} \hat{b}^{\sigma}\right)\right. \\
& +\left(\Pi^{\alpha \sigma}+3 \hat{b}^{\alpha} \hat{b}^{\sigma}\right)\left(D_{35}\left(u_{1}^{\beta} u_{2}^{\nu}\left(a_{2} \cdot u_{1}\right)+a_{2}^{\nu} u_{2 \perp}^{\beta}\right)\right. \\
& \left.+\left(D_{36} a_{1}^{\beta} a_{2 \sigma} u_{1}^{\nu}+D_{37} u_{1}^{\beta} u_{2}^{\nu}\left(a_{2 \sigma}\left(a_{1} \cdot u_{2}\right)+\gamma a_{1 \sigma}\left(a_{2} \cdot u_{1}\right)\right)-D_{22} a_{1 \sigma} a_{2}^{\beta} u_{1}^{\nu}\right)\right) \\
& +\left(\Pi^{\sigma \nu}+2 \hat{b}^{\sigma} \hat{b}^{\nu}\right)\left(-D_{27} \hat{b}_{\sigma} a_{2}^{\alpha}\left(a_{1} \cdot u_{2}\right)\left(u_{1}^{\beta}-2 \gamma u_{2}^{\beta}\right)-D_{38} \hat{b}^{\alpha} u_{1 \perp}^{\beta} a_{1 \sigma}\left(a_{2} \cdot u_{1}\right)\right. \\
& +\hat{b}_{\sigma} u_{2}^{\beta} u_{1}^{\alpha}\left(D_{28}\left(a_{1} \cdot a_{2}\right)-2 \gamma D_{27}\left(a_{1} \cdot u_{2}\right)\left(a_{2} \cdot u_{1}\right)\right)-D_{28} u_{1}^{\alpha} u_{2}^{\beta} a_{1 \sigma}\left(\hat{b} \cdot a_{2}\right) \\
& \left.+\left(\gamma^{2}-1\right) D_{38} a_{1 \sigma} a_{2}^{\alpha} \hat{b}^{\beta}+\frac{1}{2} \gamma D_{28} a_{1}^{\alpha} a_{2 \sigma} \hat{b}^{\beta}+\frac{D_{28}}{2 \gamma}\left(a_{1} \cdot u_{2}\right) \hat{b}^{\alpha} a_{2 \sigma}\left(\gamma u_{1}^{\beta}+u_{2}^{\beta}\right)\right) \\
& -D_{28}\left(\Pi^{\sigma \rho}+2 \hat{b}^{\rho} \hat{b}^{\sigma}\right) a_{1 \sigma} a_{2 \rho} \hat{b}^{\alpha} u_{1}^{\beta} u_{2}^{\nu}+D_{39} a_{1}^{\alpha} u_{1}^{\beta} u_{2}^{\nu}\left(a_{2} \cdot \hat{b}\right)-D_{28} a_{2}^{\alpha} u_{1}^{\beta} u_{2}^{\nu}\left(a_{1} \cdot \hat{b}\right) \\
& +a_{1}^{\alpha} \hat{b}^{\beta}\left(a_{2} \cdot u_{1}\right)\left(D_{16} u_{1}^{\nu}+D_{40} u_{2}^{\nu}\right)+\hat{b}^{\alpha} u_{1}^{\beta} u_{2}^{\nu}\left(D_{28}\left(a_{1} \cdot a_{2}\right)+D_{41}\left(a_{1} \cdot u_{2}\right)\left(a_{2} \cdot u_{1}\right)\right) \\
& +a_{2}^{\alpha} \hat{b}^{\beta}\left(a_{1} \cdot u_{2}\right)\left(D_{42} u_{1}^{\nu}+D_{41} u_{2}^{\nu}\right)+D_{37} a_{2}^{\alpha} u_{1}^{\beta} u_{2}^{\nu}\left(a_{1} \cdot u_{2}\right)+D_{43} a_{1}^{\alpha} u_{1}^{\beta} u_{2}^{\nu}\left(a_{2} \cdot u_{1}\right) \\
& \left.+\gamma D_{40} a_{1}^{\alpha} a_{2}^{\beta} \hat{b}^{\nu}-D_{36} a_{1}^{\alpha} a_{2}^{\beta} u_{1}^{\nu}\right] \text {, } \\
& \Delta_{a^{2}}^{(2)} S_{1}^{\mu}=\frac{\nu G^{2} M^{3}}{|b|^{2}} \epsilon_{\nu \alpha \beta}^{\mu}\left[D_{26} a_{1 \rho} a_{1 \sigma} u_{1}^{\beta} u_{2}^{\nu}\left(T^{\alpha \rho \sigma}+4 \hat{b}^{\alpha} \hat{b}^{\rho} \hat{b}^{\sigma}\right)\right. \\
& +a_{1}^{\nu} a_{1 \sigma}\left(\frac{2}{3} D_{5} u_{1}^{\beta}+D_{1} u_{2 \perp}^{\beta}\right)\left(\Pi^{\alpha \sigma}+3 \hat{b}^{\alpha} \hat{b}^{\sigma}\right) \\
& +\left(\Pi^{\nu \sigma}+2 \hat{b}^{\sigma} \hat{b}^{\nu}\right)\left(D_{28} u_{1}^{\alpha} u_{2}^{\beta}\left(a_{1}^{2} \hat{b}_{\sigma}-a_{1 \sigma}\left(\hat{b} \cdot a_{1}\right)\right)\right. \\
& +2 \gamma D_{27} \hat{b}^{\alpha} a_{1 \sigma}\left(a_{1} \cdot u_{2}\right)\left(u_{2 \perp}^{\beta}+\left(\gamma^{2}-1\right) u_{1}^{\beta}\right)-D_{27}\left(a_{1} \cdot u_{2}\right) a_{1}^{\alpha} \hat{b}_{\sigma}\left(u_{1}^{\beta}-2 \gamma u_{2}^{\beta}\right) \\
& \left.+D_{28}\left(a_{1} \cdot u_{2}\right) a_{1 \sigma} \hat{b}^{\alpha} u_{1}^{\beta}+D_{29} \hat{b}^{\beta} a_{1}^{\alpha} a_{1 \sigma}\right)+D_{28} \hat{b}^{\alpha} u_{1}^{\nu} u_{2}^{\beta} a_{1 \rho} a_{1 \sigma}\left(\Pi^{\sigma \rho}+2 \hat{b}^{\rho} \hat{b}^{\sigma}\right) \\
& +\hat{b}^{\nu} u_{1}^{\alpha} u_{2}^{\beta}\left(D_{30} a_{1}^{2}+D_{31}\left(a_{1} \cdot u_{2}\right)^{2}\right)+D_{32} a_{1}^{\nu} u_{1}^{\alpha} u_{2}^{\beta}\left(a_{1} \cdot \hat{b}\right) \\
& \left.+a_{1}^{\nu} \hat{b}^{\alpha}\left(a_{1} \cdot u_{2}\right)\left(D_{33} u_{1}^{\beta}+D_{34} u_{2}^{\beta}\right)-\frac{2}{3} D_{10} a_{1}^{\alpha} u_{1}^{\beta} u_{2}^{\nu}\left(a_{1} \cdot u_{2}\right)\right] \text {, }
\end{aligned}
$$

with the remaining $D_{i}$ 's also collected in appendix A.

\subsection{Canonical variables}

In order to apply the B2B dictionary we must also understand the map to canonical variables, in particular for the orbital angular momentum. This will be useful also to compare our results with the derivations in [122], obtained directly in terms of canonical spins. Here we follow closely the analysis put forward in $[135,136]$ (see also [41]), which we recommend for further details, while warning the reader to pay attention to the different conventions.

The canonical (or Newton-Wigner) spin constraints may be written with the aid of a background time-like four-vector, $U^{\mu}$, such that the SSC becomes, in contrast to (2.15),

$$
\mathcal{S}_{\text {can }}^{\mu \nu}\left(U_{\nu}+u_{\nu}\right)=0,
$$

for the (initial) spin and velocities. One can then search for a transformation

$$
\mathcal{S}_{\text {can }}^{\mu \nu}=\mathcal{S}^{\mu \nu}+m u^{[\mu} \delta x^{\nu]},
$$


between covariant and canonical variables, with $x_{\text {can }}^{\mu}=x^{\mu}+\delta x^{\mu}$ (obeying $\delta x \cdot u=0$ ). We proceed as follows. Firstly, we split the velocity as

$$
u^{\mu}=\hat{E} U^{\mu}+u_{\perp}^{\mu},
$$

with $\hat{E}=E / m \equiv u \cdot U$, the body's (reduced) energy in the $U$-frame. Hence, introducing the canonical spin vector as

$$
a_{\text {can }}^{\mu} \equiv \frac{1}{2 m} \epsilon_{\nu \alpha \beta}^{\mu} U^{\nu} \mathcal{S}_{\text {can }}^{\alpha \beta}
$$

we find

$$
a_{\mathrm{can}}^{\mu}=a^{\mu}+\frac{u_{\perp} \cdot a}{\hat{E}}\left(U^{\mu}+\frac{u_{\perp}^{\mu}}{\hat{E}+1}\right),
$$

for the relationship to the covariant spin four-vector, and

$$
\delta x^{\mu}=-\frac{1}{\hat{E}+1} \mathcal{S}_{\mathrm{can}}^{\mu \alpha} u_{\perp \alpha} .
$$

We now move to the two-body problem and the CoM frame, choosing the background four-vector as $U^{\nu}=\delta_{0}^{\nu}$. Using the SSC for the covariant spin we can re-write (4.19) as

$$
a_{A, \text { can }}^{0}=0, \quad \boldsymbol{a}_{A, \mathrm{can}}=\boldsymbol{a}_{A}-\frac{\boldsymbol{u}_{A, \perp} \cdot \boldsymbol{a}_{A}}{\hat{E}_{A}\left(\hat{E}_{A}+1\right)} \boldsymbol{u}_{A, \perp},
$$

for each particle. For the sake of comparison, it is also convenient to invert the relationship,

$$
\begin{aligned}
a_{A}^{0} & =\frac{\boldsymbol{u}_{A, \perp} \cdot \boldsymbol{a}_{A}}{\hat{E}_{A}}=\boldsymbol{u}_{A, \perp} \cdot \boldsymbol{a}_{A, \mathrm{can}}, \\
\boldsymbol{a}_{A} & =\boldsymbol{a}_{A, \mathrm{can}}+\frac{\boldsymbol{u}_{A, \perp} \cdot \boldsymbol{a}_{A, \mathrm{can}}}{\left(\hat{E}_{A}+1\right)} \boldsymbol{u}_{A, \perp},
\end{aligned}
$$

where the velocity is given by $\boldsymbol{u}_{A, \perp}=(-1)^{A+1} \boldsymbol{p} / m_{A}$ in the CoM frame.

Finally, using that $u_{\perp} \cdot U=0$, we also find

$$
\delta \boldsymbol{x}_{A}=\frac{\boldsymbol{u}_{A, \perp} \times \boldsymbol{a}_{\mathrm{can}}}{\left(\hat{E}_{A}+1\right)} \rightarrow \boldsymbol{b}=\boldsymbol{b}_{\mathrm{can}}-\frac{\boldsymbol{p} \times \boldsymbol{\Xi}_{\mathrm{can}}}{\left(\hat{E}_{A}+1\right)},
$$

for the change of impact parameter between covariant and canonical coordinates, where we introduced the three-vector

$$
\boldsymbol{\Xi} \equiv \sum_{A} \frac{\boldsymbol{a}_{A, \mathrm{can}}}{m_{A}\left(\hat{E}_{A}+1\right)} .
$$

From (4.21)-(4.22) it follows that the spin variables remain invariant for aligned spins, for which $\boldsymbol{p} \cdot \boldsymbol{a}=0$, and moreover do not evolve with time. In addition, the shift in (4.23) yields the relation between covariant and canonical orbital angular momentum in (3.5), which is needed for the B2B map, we implement momentarily. These transformations also allow us to compare the results reported in this paper and those in [122]. After applying (4.22)(4.23) to our results (see also eq. (2.17) in [122]) we find full agreement for the NLO spin-orbit and $\operatorname{spin}_{1}$-spin 2 momentum impulse and spin kick. ${ }^{8}$

\footnotetext{
${ }^{8}$ Notice that the condition for the spin tensor/vector in eq. (2.18) of [122] has an overall minus sign, however, they also use the (opposite) convention $\epsilon_{0123}=-1$.
} 


\subsection{Aligned-spin scattering angle}

For the case of spins aligned with the orbital angular momentum, the motion remains in the plane, and the following applies

$$
\epsilon_{\mu \nu \alpha \sigma} \hat{b}^{\mu} u_{1}^{\nu} u_{2}^{\alpha} a_{A}^{\sigma}=\sqrt{\gamma^{2}-1} a_{A}
$$

with the sign of $a_{A}\left(=\boldsymbol{a}_{A} \cdot \boldsymbol{L}\right)$ determined by the direction of the spin w.r.t. the orbital angular momentum. The scattering angle then follows from the total change of momentum in the CoM, see (2.21). The result, to $\mathcal{O}\left(G^{2}\right)$ and quadratic order in the spins, reads

$$
\begin{aligned}
& \frac{\Delta_{\left(a, a^{2}\right)} \chi}{\Gamma}=-\frac{G M}{|b|}\left(\frac{4 \gamma}{\sqrt{\gamma^{2}-1}} \frac{a_{+}}{|b|}-\frac{2 \gamma^{2}-1}{2\left(\gamma^{2}-1\right)} \frac{\left(\kappa_{+}+2\right) a_{+}^{2}+\left(\kappa_{+}-2\right) a_{-}^{2}+2 \kappa_{-} a_{-} a_{+}}{|b|^{2}}\right) \\
& -\pi\left(\frac{G M}{|b|}\right)^{2}\left(\frac{\gamma\left(5 \gamma^{2}-3\right)}{4\left(\gamma^{2}-1\right)^{3 / 2}} \frac{7 a_{+}+\delta a_{-}}{|b|}-\frac{3}{256\left(\gamma^{2}-1\right)^{2}} \frac{\lambda_{++} a_{+}^{2}+\lambda_{--} a_{-}^{2}+2 \lambda_{+-} a_{+} a_{-}}{|b|^{2}}\right)
\end{aligned}
$$

including finite-size effects, with

$$
\begin{aligned}
& \lambda_{++}=830 \gamma^{4}-876 \gamma^{2}+110+\left(35 \gamma^{4}-54 \gamma^{2}+19\right) \delta \kappa_{-}+\left(215 \gamma^{4}-222 \gamma^{2}+39\right) \kappa_{+}, \\
& \lambda_{--}=-450 \gamma^{4}+468 \gamma^{2}-82+\left(35 \gamma^{4}-54 \gamma^{2}+19\right) \delta \kappa_{-}+\left(215 \gamma^{4}-222 \gamma^{2}+39\right) \kappa_{+}, \\
& \lambda_{+-}=\left(215 \gamma^{4}-222 \gamma^{2}+39\right) \kappa_{-}+\left(\gamma^{2}-1\right)\left(70 \gamma^{2}+10+\left(35 \gamma^{2}-19\right) \delta \kappa_{+}\right) .
\end{aligned}
$$

It is straightforward to show that, for Kerr black holes, we have

$$
\begin{aligned}
\frac{\Delta_{\text {Kerr }}^{(1)} \chi}{\Gamma}= & -\frac{G M}{|b|}\left(\frac{4 \gamma}{\sqrt{\gamma^{2}-1}} \frac{a_{+}}{|b|}-\frac{2\left(2 \gamma^{2}-1\right)}{\left(\gamma^{2}-1\right)} \frac{a_{+}^{2}}{|b|^{2}}\right)+\mathcal{O}\left(a^{3}\right) \\
\frac{\Delta_{\text {Kerr }}^{(2)} \chi}{\Gamma}= & -\pi \frac{G^{2} M^{2}}{|b|^{2}}\left(\frac{\gamma\left(5 \gamma^{2}-3\right)}{4\left(\gamma^{2}-1\right)^{3 / 2}}\left(\delta \frac{a_{-}}{|b|}+7 \frac{a_{+}}{|b|}\right)-\frac{3}{64\left(\gamma^{2}-1\right)^{2}}\left[14 \delta\left(5 \gamma^{4}-6 \gamma^{2}+1\right) \frac{a_{+} a_{-}}{|b|^{2}}\right.\right. \\
& \left.\left.-\left(5 \gamma^{4}-6 \gamma^{2}+1\right) \frac{a_{-}^{2}}{|b|^{2}}+\left(315 \gamma^{4}-330 \gamma^{2}+47\right) \frac{a_{+}^{2}}{|b|^{2}}\right]\right)+\mathcal{O}\left(a^{3}\right)
\end{aligned}
$$

such that our result is consistent with [135] and the conjecture in [136], respectively.

In order to apply the B2B map, we must re-write the expression in (4.26) as an expansion in $\ell$ and $\tilde{a}_{ \pm}$, to read off the relevant PM coefficients of the scattering angle. Using the decomposition in (3.17), we find the following values

$$
\begin{aligned}
& \chi_{+}^{(2)}=\frac{\left(2 \gamma^{2}-1\right)}{\sqrt{\gamma^{2}-1}} \frac{\gamma-1}{\Gamma+1}-\frac{2 \gamma \sqrt{\gamma^{2}-1}}{\Gamma}, \\
& \chi_{-}^{(2)}=-\frac{\left(2 \gamma^{2}-1\right)}{\sqrt{\gamma^{2}-1}} \frac{(\gamma-1) \delta}{\Gamma(\Gamma+1)}, \\
& \chi_{+}^{(3)}=\frac{\pi}{4}\left(\frac{3(\gamma-1)\left(5 \gamma^{2}-1\right)}{\Gamma(\Gamma+1)}-\frac{7 \gamma\left(5 \gamma^{2}-3\right)}{2 \Gamma^{2}}\right), \\
& \chi_{-}^{(3)}=-\frac{\pi \delta}{4}\left(\frac{3(\gamma-1)\left(5 \gamma^{2}-1\right)}{\Gamma^{2}(\Gamma+1)}+\frac{\left(5 \gamma^{2}-3\right) \gamma}{2 \Gamma^{2}}\right),
\end{aligned}
$$




$$
\begin{aligned}
& \chi_{++}^{(3)}=\frac{1}{8 \Gamma^{2}}\left(\frac{8(\gamma-1)^{2}\left(2 \gamma^{2}-1\right) \Gamma^{2}}{\sqrt{\gamma^{2}-1}(\Gamma+1)^{2}}-\frac{32 \Gamma \gamma(\gamma-1) \sqrt{\gamma^{2}-1}}{\Gamma+1}+2 \sqrt{\gamma^{2}-1}\left(2 \gamma^{2}-1\right)\left(\kappa_{+}+2\right)\right), \\
& \chi_{--}^{(3)}=\frac{1}{8 \Gamma^{2}}\left(\frac{8(\gamma-1)^{2}\left(2 \gamma^{2}-1\right) \delta^{2}}{\sqrt{\gamma^{2}-1}(\Gamma+1)^{2}}+2 \sqrt{\gamma^{2}-1}\left(2 \gamma^{2}-1\right)\left(\kappa_{+}-2\right)\right), \\
& \begin{array}{c}
\chi_{-+}^{(3)}=\chi_{+-}^{(3)}=\frac{1}{\Gamma^{2}}\left(\frac{\left(2 \gamma^{2}-1\right)(\gamma-1)^{2} \delta}{\sqrt{\gamma^{2}-1}(\Gamma+1)^{2}}+\frac{16(2 \gamma+1)(\gamma-1)^{2} \delta}{\sqrt{\gamma^{2}-1}(\Gamma+1)}+\frac{1}{4} \sqrt{\gamma^{2}-1}\left(2 \gamma^{2}-1\right) \kappa_{-}\right), \\
\chi_{++}^{(4)}=\frac{3 \pi}{512 \Gamma^{3}}\left(\frac{192 \Gamma^{2}(\gamma-1)^{2}\left(5 \gamma^{2}-1\right)}{(\Gamma+1)^{2}}-\frac{448 \Gamma \gamma(\gamma-1)\left(5 \gamma^{2}-3\right)}{(\Gamma+1)}+830 \gamma^{4}-876 \gamma^{2}+110\right. \\
\left.\quad+\delta \kappa_{-}\left(35 \gamma^{4}-54 \gamma^{2}+19\right)+\kappa_{+}\left(215 \gamma^{4}-222 \gamma^{2}+39\right)\right), \\
\chi_{--}^{(4)}=\frac{3 \pi}{512 \Gamma^{3}}\left(\frac{192 \delta^{2}(\gamma-1)^{2}\left(5 \gamma^{2}-1\right)}{(\Gamma+1)^{2}}+\frac{64 \delta^{2} \gamma(\gamma-1)\left(5 \gamma^{2}-3\right)}{(\Gamma+1)}-450 \gamma^{4}+468 \gamma^{2}-82\right. \\
\left.\quad+\delta \kappa_{-}\left(35 \gamma^{4}-54 \gamma^{2}+19\right)+\kappa_{+}\left(215 \gamma^{4}-222 \gamma^{2}+39\right)\right), \\
\chi_{-+}^{(4)}=\chi_{+-}^{(4)}=\frac{3 \pi}{512 \Gamma^{3}}\left(\frac{192\left(5 \gamma^{2}-1\right)(\gamma-1)^{2} \delta}{(\Gamma+1)^{2}}+\frac{64\left(5 \gamma^{4}+10 \gamma^{3}-24 \gamma^{2}+6 \gamma+3\right) \delta}{\Gamma+1}\right. \\
\left.-2(\gamma-1)\left(45 \gamma^{3}-35 \gamma^{2}-53 \gamma-5\right) \delta+\left(215 \gamma^{4}-222 \gamma^{2}+39\right) \kappa_{-}+\left(\gamma^{2}-1\right)\left(35 \gamma^{2}-19\right) \delta \kappa_{+}\right) .
\end{array}
\end{aligned}
$$

\section{Bound states to 2PM: linear and bilinear (aligned) spin effects}

Once the scattering angle is computed the radial action for the bound problem follows, via analytic continuation. Crucially, the relationship between $b$ and $\ell$ in (3.5) also introduces spin effects. This forces us to keep not only spin-dependent terms but also the spinindependent corrections, computed in [82]. From the radial action it is straightforward to derive the gravitational observables through differentiation. In what follows we illustrate the procedure to $2 \mathrm{PM}$ order. We also discuss how to incorporate the extra terms needed to complete the NLO contributions to the binding energy linear and bilinear in spin to 3PN order. Finally, we provide the coefficients in the CoM momentum to $\mathcal{O}\left(G^{2}\right)$, and all orders in velocity.

\subsection{Radial action}

From the terms in (4.30)-(4.38), only those which are even in the total angular momentum survive the B2B map, yielding for the (bound) radial action to 2PM order:

$$
i_{r}^{2 \mathrm{PM}}\left(\mathcal{E}, \ell, \tilde{a}_{ \pm}\right)=-\ell+\frac{2 \gamma^{2}-1}{\sqrt{1-\gamma^{2}}}+\frac{3}{4 \ell} \frac{5 \gamma^{2}-1}{\Gamma}+\frac{1}{\pi} \sum_{A= \pm} \chi_{A}^{(3)}(\gamma) \frac{\tilde{a}_{A}}{\ell^{2}}+\frac{2}{3 \pi} \sum_{\{A, B\}= \pm} \chi_{A B}^{(4)}(\gamma) \frac{\tilde{a}_{A} \tilde{a}_{B}}{\ell^{3}}
$$

after adding the results in [82] for the spin-independent terms. The reader will immediately notice that the analytic continuation to negative binding energies $(\gamma<1)$ follows smoothly. While the expression in (5.1) allows us to compute observables, incorporating an infinite 
series of velocity corrections at a given order in $1 / \ell$, the fact that we truncate the radial action in the PM expansion prevents us from having direct access to the information needed to consistently obtain the PN effects associated with our PM results, for example the binding energy. This, however, is easily remediated by using the $f_{n}$ 's in (3.6) and the representation in (3.9), yielding a consistent PN-truncation in higher orders terms. As in the non-spinning case, we found in section 3.3 that the coefficients of the CoM impetus can be obtained directly from the scattering angle, albeit in a more intricate fashion. We display their full expressions shortly. Once these are known, only the static limit is needed to add the terms required to complete the knowledge of the dynamics to NLO in the PN expansion. Using (3.10), we have

$$
i_{r}^{2 \mathrm{PM} / 3 \mathrm{PN}}=i_{r}^{2 \mathrm{PM}}+\Delta i_{r}^{3 \mathrm{PN}},
$$

with

$$
\begin{aligned}
\Delta i_{r}^{3 \mathrm{PN}}= & \left(\frac{1}{2 \pi} \sum_{A= \pm} \chi_{A}^{(5)}(\gamma) \frac{\tilde{a}_{A}}{\ell^{4}}+\frac{2}{5 \pi} \sum_{\{A, B\}= \pm} \chi_{A B}^{(6)}(\gamma) \frac{\tilde{a}_{A} \tilde{a}_{B}}{\ell^{5}}\right)_{\gamma \rightarrow 1} \\
= & \left(\frac{3 \hat{p}_{\infty}^{6}}{16 \ell^{4}} \sum_{A= \pm} \tilde{a}_{A}\left(2 f_{1}^{0} f_{2}^{0} f_{3}^{A}+\left(f_{1}^{0}\right)^{2} f_{4}^{A}\right)+\frac{3 \hat{p}_{\infty}^{6}}{16 \ell^{5}} \sum_{\{A, B\}= \pm} \tilde{a}_{A} \tilde{a}_{B}\left(f_{4}^{A B}\left(f_{1}^{0}\right)^{2}+2 f_{3}^{A B} f_{1}^{0} f_{2}^{0}\right)\right. \\
& \left.+\sum_{\{A, B\}= \pm} \frac{15 \hat{p}_{\infty}^{8}}{64 \ell^{5}}\left(f_{1}^{0}\right)^{2} f_{3}^{A} f_{3}^{B} \tilde{a}_{A} \tilde{a}_{B}\right)_{\gamma \rightarrow 1}
\end{aligned}
$$

where we only keep the leading PN corrections. As discussed in [80-84], the remaining terms in (3.10) have fewer factors of $f_{i}$ 's, thus scaling with additional powers of $\hat{p}_{\infty}^{2} \sim \mathcal{E}$ relative to the ones displayed, and therefore contributing at higher PN order. (This is a consequence of the fact that the impetus in the CoM has well-defined static limit, so that $f_{i} \sim 1 / \hat{p}_{\infty}^{2}$.)

\subsection{Observables}

\subsubsection{Periastron advance}

The periastron advance follows from the radial action via

$$
\frac{\Delta \Phi}{2 \pi}=-\frac{\partial}{\partial \ell}\left(i_{r}+\ell\right) \text {. }
$$

However, this is obviously equivalent to the condition in (3.1), which we used to build the radial action. Using (4.26), translated to orbital angular momentum space, we find to $\mathcal{O}\left(G^{2}\right)$

$$
\begin{aligned}
\frac{\Delta \Phi}{2 \pi}= & \frac{3\left(5 \gamma^{2}-1\right)}{4 \Gamma} \frac{1}{\ell^{2}}+\left[\frac{6}{\Gamma+1}\left(5 \gamma^{2}-1\right)(\gamma-1)\left(\Gamma \tilde{a}_{+}-\delta \tilde{a}_{-}\right)-\gamma\left(5 \gamma^{2}-3\right)\left(\delta \tilde{a}_{-}+7 \tilde{a}_{+}\right)\right] \frac{1}{4 \Gamma^{2} \ell^{3}} \\
& +\left[-\frac{64 \gamma\left(5 \gamma^{2}-3\right)(\gamma-1)}{\Gamma+1}\left(\delta \tilde{a}_{-}+7 \tilde{a}_{+}\right)\left(\Gamma \tilde{a}_{+}-\delta \tilde{a}_{-}\right)\right. \\
& \left.+\frac{192\left(5 \gamma^{2}-1\right)(\gamma-1)^{2}}{(\Gamma+1)^{2}}\left(\Gamma \tilde{a}_{+}-\delta \tilde{a}_{-}\right)^{2}+\lambda_{--} \tilde{a}_{-}^{2}+2 \lambda_{+-} \tilde{a}_{+} \tilde{a}_{-}+\lambda_{++} \tilde{a}_{+}^{2}\right] \frac{3}{256 \Gamma^{3} \ell^{4}}+\cdots
\end{aligned}
$$


where the $\lambda_{A B}$ coefficients are given in (4.27). In order to compare with the PN literature, we expand it in powers of $\epsilon \equiv-2 \mathcal{E}$, yielding

$$
\begin{aligned}
\frac{\Delta \Phi(\ell, a, \epsilon)}{2 \pi}=[3+ & \left.\frac{3(2 \nu-5)}{4} \epsilon+\frac{3\left(5-5 \nu+4 \nu^{2}\right)}{16} \epsilon^{2}\right] \frac{1}{\ell^{2}} \\
+ & {\left[-\frac{7 \tilde{a}_{+}+\delta \tilde{a}_{-}}{2}-\frac{(\nu-6) \delta \tilde{a}_{-}+(7 \nu-18) \tilde{a}_{+}}{2} \epsilon\right.} \\
& \left.\quad-\frac{3\left(\left(15-14 \nu+2 \nu^{2}\right) \delta \tilde{a}_{-}+\left(25-38 \nu+14 \nu^{2}\right) \tilde{a}_{+}\right)}{16} \epsilon^{2}\right] \frac{1}{\ell^{3}} \\
+ & {\left[\frac{3}{8}\left(\tilde{a}_{-}^{2}\left(\kappa_{+}-2\right)+2 \tilde{a}_{+} \tilde{a}_{-} \kappa_{-}+\tilde{a}_{+}^{2}\left(\kappa_{+}+2\right)\right)\right.} \\
& \quad-\frac{3}{16} \epsilon\left(\tilde{a}_{-}^{2}\left(\delta \kappa_{-}+\kappa_{+}(13-3 \nu)-2 \nu-25\right)+2 \tilde{a}_{+} \tilde{a}_{-}\left(\kappa_{-}(13-3 \nu)+\delta\left(\kappa_{+}+11\right)\right)\right. \\
& \left.\left.+\tilde{a}_{+}^{2}\left(\delta \kappa_{-}+\kappa_{+}(13-3 \nu)-6 \nu+35\right)\right)+\cdots\right] \frac{1}{\ell^{4}}+\cdots
\end{aligned}
$$

The comparison with the PN result given in eq. (33) of [145] thus gives perfect agreement, including finite-size effects. ${ }^{9}$ Yet, the expression in (5.5) contains all orders in $\epsilon$, at $\mathcal{O}\left(a / \ell^{3}\right)$ and $\mathcal{O}\left(a^{2} / \ell^{4}\right)$, extending the results in [81] at quadratic order in spins.

\subsubsection{Binding energy}

The binding energy for circular orbits can be computed in different ways. One option is to get the value of the orbital angular momentum as a function of the energy, $\ell_{c}\left(\mathcal{E}_{c}, a_{ \pm}\right)$, from the condition $i_{r}\left(\ell_{c}\left(\mathcal{E}_{c}, a_{ \pm}\right), \mathcal{E}_{c}, a_{ \pm}\right)=0$. (Alternatively we can use the condition $r_{+}=r_{-}$ for the roots of (3.6) in a circular orbits [80].) From the orbital angular momentum we obtain the orbital frequency, $\Omega_{c}$, via the first-law [146]

$$
x \equiv\left(G M \Omega_{c}\right)^{2 / 3}=\left(\frac{d \ell_{c}}{d \mathcal{E}_{c}}\right)^{-2 / 3},
$$

from which we extract the relationship $\mathcal{E}_{c}\left(x, a_{ \pm}\right)$for circular orbits. Using the expression in (5.2), which includes a few terms at higher orders in $1 / \ell$ needed to account for all the contributions to NLO in the PN expansion, we find (recall $\epsilon=-2 \mathcal{E}$ )

$$
\begin{aligned}
\epsilon_{c}=x & -\frac{x^{2}}{12}(\nu+9)+x^{5 / 2}\left(\frac{1}{3}\left(\delta \tilde{a}_{-}+7 \tilde{a}_{+}\right)+\frac{x}{18}\left[(99-61 \nu) \tilde{a}_{+}-(\nu-45) \delta \tilde{a}_{-}\right]\right) \\
+ & \frac{1}{6} x^{3}\left[-\left(\kappa_{+}+2\right) \tilde{a}_{+}^{2}-\left(\kappa_{+}-2\right) \tilde{a}_{-}^{2}-2 \kappa_{-} \tilde{a}_{-} \tilde{a}_{+}\right] \\
+ & \frac{5}{72} x^{4}\left[\left(6(\nu-5) \kappa_{-}-4\left(3 \kappa_{+}+5\right) \delta\right) \tilde{a}_{-} \tilde{a}_{+}+\left(32-6 \delta \kappa_{-}+10 \nu+3(\nu-5) \kappa_{+}\right) \tilde{a}_{-}^{2}\right. \\
& \left.+\left(20-6 \delta \kappa_{-}+6 \nu+3(\nu-5) \kappa_{+}\right) \tilde{a}_{+}^{2}\right]+\cdots,
\end{aligned}
$$

${ }^{9}$ The error in [145], first pointed out in [81], turns out to be a mere factor of 2 when looking at their result with $C_{Q} \neq 1$. The correct value is given by replacing $\left(7-\frac{33 \nu}{4}+3 \nu^{2}\right) \rightarrow\left(7-\frac{33 \nu}{2}+3 \nu^{2}\right)$ in their eq. (33). 
to $3 \mathrm{PN}$ and quadratic order in spins. After translating between various conventions, this agrees with the known value in the literature [27, 35-41, 50, 85].

\subsection{Center-of-mass momentum}

The PM coefficients of the impetus in (3.6), written using in the decomposition in (3.16), follow by inverting the relations in section 3.3. We obtain

$$
\begin{aligned}
f_{3}^{A} & =\frac{2 \chi_{A}^{(2)}}{\hat{p}_{\infty}^{3}}, & f_{3}^{A B} & =\frac{\chi_{A B}^{(3)}}{\hat{p}_{\infty}^{3}} \\
f_{4}^{A} & =\frac{4}{\hat{p}_{\infty}^{4}}\left(\frac{\chi_{A}^{(3)}}{\pi}-\chi_{0}^{(1)} \chi_{A}^{(2)}\right), & f_{4}^{A B} & =\frac{2}{\hat{p}_{\infty}^{4}}\left(4 \frac{\chi_{A B}^{(4)}}{3 \pi}-\chi_{0}^{(1)} \chi_{A B}^{(3)}-\frac{3}{2} \chi_{A}^{(2)} \chi_{B}^{(2)}\right),
\end{aligned}
$$

where we used $f_{1}^{0}=\frac{2}{\hat{p}_{\infty}} \chi_{0}^{(1)}[80]$. Then, from the data collected in (4.30)-(4.38), we find

$$
\begin{aligned}
f_{3}^{(+)}= & -\frac{4 \gamma \Gamma^{2}}{\gamma^{2}-1}+\frac{2(\gamma-1)\left(2 \gamma^{2}-1\right) \Gamma^{3}}{\left(\gamma^{2}-1\right)^{2}(\Gamma+1)} \\
f_{3}^{(-)}= & -\frac{2\left(2 \gamma^{2}-1\right) \Gamma^{2} \delta}{(\gamma-1)(\gamma+1)^{2}(\Gamma+1)}, \\
f_{3}^{(++)}= & \frac{\left(2 \gamma^{2}-1\right) \Gamma^{3}}{(\gamma+1)^{2}(\Gamma+1)^{2}}-\frac{4 \gamma \Gamma^{2}}{(\gamma+1)(\Gamma+1)}+\frac{\left(2 \gamma^{2}-1\right) \Gamma\left(\kappa_{+}+2\right)}{4\left(\gamma^{2}-1\right)} \\
f_{3}^{(--)}= & \frac{\left(2 \gamma^{2}-1\right) \Gamma \delta^{2}}{(\gamma+1)^{2}(\Gamma+1)^{2}}+\frac{\left(2 \gamma^{2}-1\right) \Gamma\left(\kappa_{+}-2\right)}{4\left(\gamma^{2}-1\right)} \\
f_{3}^{(+-)}= & f_{3}^{(-+)}=\frac{2 \gamma \Gamma \delta}{(\gamma+1)(\Gamma+1)}-\frac{\left(2 \gamma^{2}-1\right) \Gamma^{2} \delta}{(\gamma+1)^{2}(\Gamma+1)^{2}}+\frac{\left(2 \gamma^{2}-1\right) \Gamma \kappa_{-}}{4\left(\gamma^{2}-1\right)} \\
f_{4}^{(+)}= & \frac{8 \gamma\left(2 \gamma^{2}-1\right) \Gamma^{3}}{\left(\gamma^{2}-1\right)^{2}}-\frac{4\left(2 \gamma^{2}-1\right)^{2} \Gamma^{4}}{(\gamma-1)^{2}(\gamma+1)^{3}(\Gamma+1)}-\frac{7 \gamma\left(5 \gamma^{2}-3\right) \Gamma^{2}}{2\left(\gamma^{2}-1\right)^{2}} \\
& +\frac{3\left(5 \gamma^{2}-1\right) \Gamma^{3}}{(\gamma-1)(\gamma+1)^{2}(\Gamma+1)}, \\
f_{4}^{(-)}= & \frac{4\left(2 \gamma^{2}-1\right)^{2} \Gamma^{3} \delta}{(\gamma-1)^{2}(\gamma+1)^{3}(\Gamma+1)}-\frac{\gamma\left(5 \gamma^{2}-3\right) \Gamma^{2} \delta}{2\left(\gamma^{2}-1\right)^{2}}-\frac{3\left(5 \gamma^{2}-1\right) \Gamma^{2} \delta}{(\gamma-1)(\gamma+1)^{2}(\Gamma+1)} \\
f_{4}^{(++)}= & -\frac{5\left(2 \gamma^{2}-1\right)^{2} \Gamma^{4}}{(\gamma-1)(\gamma+1)^{3}(\Gamma+1)^{2}}+\frac{20 \gamma\left(2 \gamma^{2}-1\right) \Gamma^{3}}{(\gamma-1)(\gamma+1)^{2}(\Gamma+1)}+\frac{3\left(5 \gamma^{2}-1\right) \Gamma^{3}}{(\gamma+1)^{2}(\Gamma+1)^{2}} \\
& -\frac{\left(2 \gamma^{2}-1\right)^{2} \Gamma^{2}(\kappa++2)}{2\left(\gamma^{2}-1\right)^{2}}-\frac{12 \gamma^{2} \Gamma^{2}}{\gamma^{2}-1} \frac{7 \gamma\left(5 \gamma^{2}-3\right) \Gamma^{2}}{(\gamma-1)(\gamma+1)^{2}(\Gamma+1)} \\
& \frac{\left(35 \gamma^{2}-19\right) \Gamma \delta \kappa_{-}}{64\left(\gamma^{2}-1\right)} \frac{\left(215 \gamma^{4}-222 \gamma^{2}+39\right) \Gamma \kappa_{+}}{64} \frac{\left(415 \gamma^{4}-438 \gamma^{2}+55\right) \Gamma}{32\left(\gamma^{2}-1\right)^{2}}
\end{aligned}
$$




$$
\begin{aligned}
f_{4}^{(--)}= & -\frac{5\left(2 \gamma^{2}-1\right)^{2} \Gamma^{2} \delta^{2}}{(\gamma-1)(\gamma+1)^{3}(\Gamma+1)^{2}}-\frac{\left(2 \gamma^{2}-1\right)^{2} \Gamma^{2}\left(\kappa_{+}-2\right)}{2\left(\gamma^{2}-1\right)^{2}}+\frac{\gamma\left(5 \gamma^{2}-3\right) \Gamma \delta^{2}}{(\gamma-1)(\gamma+1)^{2}(\Gamma+1)} \\
& +\frac{3\left(5 \gamma^{2}-1\right) \Gamma \delta^{2}}{(\gamma+1)^{2}(\Gamma+1)^{2}}+\frac{\left(35 \gamma^{2}-19\right) \Gamma \delta \kappa_{-}}{64\left(\gamma^{2}-1\right)}+\frac{\left(215 \gamma^{4}-222 \gamma^{2}+39\right) \Gamma \kappa_{+}}{64\left(\gamma^{2}-1\right)^{2}} \\
& -\frac{\left(225 \gamma^{4}-234 \gamma^{2}+41\right) \Gamma}{32\left(\gamma^{2}-1\right)^{2}}, \\
f_{4}^{(+-)}= & f_{4}^{(-+)}=\frac{5\left(2 \gamma^{2}-1\right)^{2} \Gamma^{3} \delta}{(\gamma-1)(\gamma+1)^{3}(\Gamma+1)^{2}}-\frac{10 \gamma\left(2 \gamma^{2}-1\right) \Gamma^{2} \delta}{(\gamma-1)(\gamma+1)^{2}(\Gamma+1)}-\frac{3\left(5 \gamma^{2}-1\right) \Gamma^{2} \delta}{(\gamma+1)^{2}(\Gamma+1)^{2}} \\
& -\frac{\left(2 \gamma^{2}-1\right)^{2} \Gamma^{2} \kappa_{-}}{2\left(\gamma^{2}-1\right)^{2}}+\frac{\left(35 \gamma^{2}-19\right) \Gamma \delta \kappa_{+}}{64\left(\gamma^{2}-1\right)}-\frac{\gamma\left(5 \gamma^{2}-3\right)(\Gamma-7) \Gamma \delta}{2(\gamma-1)(\gamma+1)^{2}(\Gamma+1)} \\
& +\frac{5 \Gamma\left(7 \gamma^{2}+1\right) \delta}{32\left(\gamma^{2}-1\right)}+\frac{\left(215 \gamma^{4}-222 \gamma^{2}+39\right) \Gamma \kappa_{-}}{64\left(\gamma^{2}-1\right)^{2}} .
\end{aligned}
$$

The expansion of the CoM momentum can then be analytically continued to negative binding energies $(\gamma<1)$, yielding a local effective description of the dynamics in a quasiisotropic gauge, that may be used to construct the Hamiltonian. (Alternatively, one can derive it from the radial action using Delaunay variables, e.g. [78].) However, as emphasized in $[80,81]$ and clearly shown in this paper, from the point of view of the scattering angle, radial action and B2B map (as well as the amplitude through the impetus formula [80]) the $f_{n}$ 's are the most natural variables. Therefore, they are preferable to the - much more cumbersome - PM coefficients of the Hamiltonian, which we refrain from displaying here.

\section{Discussion \& outlook}

Building on the EFT approach developed in [30, 31, 37, 38, 41], in this paper we have extended the PM framework introduced in [82] to incorporate spin effects. We then used the formalism to compute the NLO momentum and spin impulses with generic initial conditions and to quadratic order in the spins, including for the first time finite-size effects beyond leading order. Afterwards we considered aligned-spin configurations and derived the scattering angle, which we used to construct the bound radial action via the B2B correspondence. The latter allows us to compute all the gravitational observables for elliptic-like orbits. As a notable example, we obtained the periastron advance to $\mathcal{O}\left(G^{2}\right)$ and all orders in velocity. We also computed the linear and bilinear in spin contributions to the binding energy for circular orbits to $3 \mathrm{PN}$ order. In addition, we derived the CoM momentum (or impetus) in a quasi-isotropic gauge, from which one can readily obtain the EoM (or the Hamiltonian) to 2PM order, if so desired. Our results are in perfect agreement with the known literature, notably spin-orbit and $\operatorname{spin}_{1}$-spin ${ }_{2}$ effects to NLO in the PM expansion in [122], while the $\operatorname{spin}_{1(2)}{ }^{-\operatorname{spin}_{1(2)}}$ contributions for generic compact bodies are computed here for the first time. We also find agreement with the conjectured value for the 2PM aligned-spin scattering angle of Kerr black holes [136].

In order to perform the comparison with the findings using scattering amplitudes in [122], we have translated our covariant results into canonical variables. The latter 
have the advantage of furnishing a canonical algebra involving only a spin three-vector, yet the Lorentz covariance of the results is hidden in somewhat cumbersome vectorial expressions. In contrast, in the former the results are not only manifestly covariant, by construction, but also remarkably compact when written in terms of four-dimensional vectors, as displayed here. In fact, due to the conservation of the $\operatorname{SSC}\left(S_{\mu} p^{\mu}=0\right)$ upon evolution, both the spin and momentum rotate in spacetime in the same fashion [135]. This implies the simple structure

$$
\Delta p^{\mu}=m \delta \Lambda_{\alpha}^{\mu} u^{\alpha}, \Delta S^{\mu}=m \delta \Lambda_{\alpha}^{\mu} a^{\alpha},
$$

with $\delta \Lambda_{\mu \nu}=-\delta \Lambda_{\nu \mu}$, must hold for both impulses. As shown in [135], the form of the $\delta \Lambda_{\mu \nu}$ matrix can be easily found at $1 \mathrm{PM}$ order in terms of a four-vector, $Z_{\mu}$, and the velocity $u_{\mu}$, obeying the condition $Z \cdot u=0$. This representation is the basis for the " $b \rightarrow b+i a$ " shift which lies at the heart of the (complex) transformation introduced in [147] connecting Schwarzschild and Kerr solutions, (see also [109]). ${ }^{10}$ At NLO, however, the construction of the $\delta \Lambda_{\mu \nu}$ matrix is less straightforward, mainly due to the new directions in which a non-zero impulse appears. For the case of non-spinning bodies, the task is relatively simple and the rotation can be easily written down incorporating the impulse in the $u^{\mu}$ direction. However, when spin effects are included, the form of the transformation turns out to be much more involved, begging instead for a more convenient basis to decompose the impulses. Without spin, such basis exists, by combining the $b^{\mu}$ and $u^{\mu}$ impulses into a spacelike vector, and it is directly connected to the eikonal representation. It would be interesting to perform the same manipulations for the case of spinning bodies, in particular in light of the remarkable algebraic structure involving the eikonal phase recently discovered in [122].

Another interesting area for further study is the possibility, for the special case of Kerr black holes, to promote the worldline effective theory into a worldsheet, as advocated in [133]. The motivation is also built on the " $x+i a$ " shift [147], which suggests extending the worldline action into one more dimension. This is not entirely surprising, after all the covariant SSC implies a non-commutative (Dirac) algebra for the position, $\left\{\boldsymbol{x}^{i}, \boldsymbol{x}^{j}\right\}_{\mathrm{DB}} \simeq \epsilon^{i j k} \boldsymbol{a}^{k} / m$, e.g. [31, 88], hinting at the extendedness of spinning particles. The worldsheet idea is also rooted on the fact that, not only the quadrupole [37], but all of the worldline (Wilson) coefficients obey $C_{E S^{2 n}}=1$ (and similarly with the magnetic terms) when matching to the multipole moments of a Kerr black hole [133]. This observation allows one to resume all the derivatively coupled higher-derivative terms in the effective action, which then exponentiate into a translation operation, $e^{i a \cdot \partial}$, that is directly linked to a complex coordinate shift. However, because of the equivalence principle, finite-size effects start with two derivatives (with the 1 and $a \partial / 2$ terms already appropriated by the mass and spin) yielding the structure $\left(e^{x}-1\right) / x=\int_{0}^{1} d \lambda e^{\lambda x}$, which naturally allows one to introduce a two-dimensional integral for the effective action. Furthermore, it turns out to be convenient to introduce a spinor-helicity representation [133] (see [154] for other possible routes). Hence, armed with an action incorporating all of the (self-induced) finite-size

\footnotetext{
${ }^{10}$ This transformation (applied to perturbations of the background) may also play a key role in understanding the vanishing of (static) tidal response for rotating black holes [61, 148-150] following the pattern observed in Schwarzschild [151-153] (http://www.youtube.com/watch?v=v9fvAohXD8g\&t=45m15s).
} 
effects at once, we could then set up the EFT formalism described in this paper, uplifted to a worldsheet Routhian, to compute the momentum and spin impulses for Kerr black holes without having to introduce the curvature terms representing finite-size effects. This possibility is currently under investigation.

Yet one more aspect of the framework which deserves more attention is the generalization of the B2B correspondence to the case of non-aligned spins. As shown in [81], the planar B2B map can be extended to spinning bodies by performing an analytic continuation

in the total (canonical) angular momentum. However, when $\dot{\boldsymbol{L}}=-\dot{\boldsymbol{S}} \neq 0$, the precession of the plane complicates matters. Moreover, only canonical variables may be associated through the B2B dictionary, which requires also transforming the spin variables when we allow for non-planar dynamics. In principle, we could consider the periastron shift in the instantaneous plane or, more likely, orbital averages over a period. For instance, because the orbital elements are related via analytic continuation - with aligned or zero spins the B2B map yields a relationship between the total radiated energy in a scattering process and the integrated power over an orbit, via analytic continuation (see footnote 5). Hence, we may expect a similar situation once we include the precession of the orbital plane, thus retaining a link between the total impulse in momentum and an averaged periastron advance. There is as well the total spin kick to be considered, and the associated change in orbital angular momentum. Provided a relationship between the orbital elements still holds, we may expect an analogous connection to the integrated change over an orbit for the bound case. Another interesting venue is to explore the modification of the first-law to the case of spinning bodies, e.g. [155], which would allow us to compute the precession frequency once the non-planar B2B map is obtained. We will return to these issues in future work. Finally, since the master integrals to $\mathrm{N}^{2} \mathrm{LO}$ are known [83], the formalism is ready to march forward in the PM expansion. The computation of spin effects to $\mathcal{O}\left(G^{3}\right)$ is currently underway.

Note added. While the results of this project were prepared for submission we learned of the concurrent work of [156], which also computed finite-size contributions to the NLO impulses via the methods discussed in [122]. After transforming between the different variables (as discussed in section 4.3) their results agree with ours. We thank the authors of [156] for confirming the perfect match with us.

\section{Acknowledgments}

We thank Gregor Kälin and Justin Vines for helpful discussions. We also thank Andres Luna and Chia-Hsien Shen for useful exchanges on the results in [122]. This work is supported by the ERC Consolidator Grant "Precision Gravity: From the LHC to LISA," provided by the European Research Council (ERC) under the European Union's H2020 research and innovation programme, grant No. 817791. Our work is also supported by the DFG under Germany's Excellence Strategy 'Quantum Universe' (No. 390833306). We acknowledge extensive use of the xAct packages (www.xact.es). 


\section{A Supplemental material}

\section{A.1 Trajectories}

An important element in the computation of the NLO impulses is iteration of the 1PM EoM into the tree-level Routhian. For completeness, we provide here the trajectories for the position and spin to $1 \mathrm{PM}$ order. To simplify the notation we always refer to the dynamics of particle 1 and do not include the mirror images. The contribution from the latter, as well as the corresponding deflection for the second particle, can be derived as explained in $[82]$.

\section{A.1.1 Velocity \& position}

The velocity correction at linear order in spin is given by,

$$
\begin{aligned}
\delta_{S_{1}}^{(1)} v_{1}^{\mu}\left(\tau_{1}\right)=\frac{-i m_{2}}{8 M_{\mathrm{Pl}}^{2} m_{1}} \int_{k} & \frac{\hat{\delta}\left(k \cdot u_{2}\right)}{k^{2}\left(k \cdot u_{1}-i \epsilon\right)} e^{i k \cdot b} e^{i\left(k \cdot u_{1}-i \epsilon\right) \tau_{1}} \\
& \times k^{\alpha}\left(k^{\nu} u_{1 \alpha}\left(\mathcal{S}_{1}{ }^{\mu}{ }_{\nu}+2 u_{2}{ }^{\beta} u_{2}{ }^{\mu} \mathcal{S}_{1 \nu \beta}\right)-2 \gamma k^{\mu} u_{2}{ }^{\nu} \mathcal{S}_{1 \alpha \nu}\right),
\end{aligned}
$$

and one time integration yields the position correction

$$
\begin{aligned}
\delta_{S_{1}}^{(1)} x_{1}^{\mu}\left(\tau_{1}\right)=\frac{-m_{2}}{8 M_{\mathrm{Pl}}^{2} m_{1}} \int_{k} & \frac{\hat{\delta}\left(k \cdot u_{2}\right)}{k^{2}\left(k \cdot u_{1}-i \epsilon\right)^{2}} e^{i k \cdot b} e^{i\left(k \cdot u_{1}-i \epsilon\right) \tau_{1}} \\
& \times k^{\alpha}\left(k^{\nu} u_{1 \alpha}\left(\mathcal{S}_{1}{ }^{\mu}{ }_{\nu}+2 u_{2}{ }^{\beta} u_{2}{ }^{\mu} \mathcal{S}_{1 \nu \beta}\right)-2 \gamma k^{\mu} u_{2}{ }^{\nu} \mathcal{S}_{1 \alpha \nu}\right) .
\end{aligned}
$$

At quadratic order in the spin we have several contributions. For the term proportional to the SSC in (2.8) we find

$$
\begin{aligned}
\delta_{R S_{1} S_{1}}^{(1)} v_{1}^{\mu}\left(\tau_{1}\right)=\frac{-m_{2}}{8 M_{\mathrm{Pl}}^{2} m_{1}^{2}} \int_{k} \frac{\hat{\delta}\left(k \cdot u_{2}\right)}{k^{2}} e^{i k \cdot b} e^{i\left(k \cdot u_{1}-i \epsilon\right) \tau_{1}}\left[k^{\nu}\left(k \cdot u_{1}\right) \mathcal{S}_{1}{ }^{\mu \beta} \mathcal{S}_{1 \nu \beta}\right. \\
\left.+2 \gamma k^{\nu} \mathcal{S}_{1}{ }^{\mu}{ }_{\nu}\left(k^{\alpha} u_{2}{ }^{\beta} \mathcal{S}_{1 \alpha \beta}\right)-2 u_{2}{ }^{\nu}\left(k \cdot u_{1}\right) \mathcal{S}_{1}{ }^{\mu}{ }_{\nu}\left(k^{\alpha} u_{2}{ }^{\beta} \mathcal{S}_{1 \alpha \beta}\right)\right],
\end{aligned}
$$

and

$$
\begin{aligned}
\delta_{R S_{1} S_{1}}^{(1)} x_{1}^{\mu}\left(\tau_{1}\right)=\frac{i m_{2}}{8 M_{\mathrm{Pl}}^{2} m_{1}^{2}} \int_{k} \frac{\hat{\delta}\left(k \cdot u_{2}\right)}{k^{2}\left(k \cdot u_{1}-i \epsilon\right)} e^{i k \cdot b} e^{i\left(k \cdot u_{1}-i \epsilon\right) \tau_{1}}\left[k^{\nu}\left(k \cdot u_{1}\right) \mathcal{S}_{1}{ }^{\mu \beta} \mathcal{S}_{1 \nu \beta}\right. \\
\left.+2 \gamma k^{\nu} \mathcal{S}_{1}{ }^{\mu}{ }_{\nu}\left(k^{\alpha} u_{2}{ }^{\beta} \mathcal{S}_{1 \alpha \beta}\right)-2 u_{2}{ }^{\nu}\left(k \cdot u_{1}\right) \mathcal{S}_{1}{ }^{\mu}{ }_{\nu}\left(k^{\alpha} u_{2}{ }^{\beta} \mathcal{S}_{1 \alpha \beta}\right)\right],
\end{aligned}
$$

whereas from the finite-size effects we arrive at

$$
\begin{aligned}
\delta_{E S_{1}^{2}}^{(1)} v_{1}^{\mu}\left(\tau_{1}\right)= & \frac{m_{2}}{16 M_{\mathrm{Pl}}^{2} m_{1}^{2}} \int_{k} \frac{\hat{\delta}\left(k \cdot u_{2}\right) k^{\alpha} k^{\nu}}{k^{2}\left(k \cdot u_{1}-i \epsilon\right)} e^{i k \cdot b} e^{i\left(k \cdot u_{1}-i \epsilon\right) \tau_{1}} \\
& \times\left[k^{\mu}\left(2 \gamma^{2}-1\right) \mathcal{S}_{1 \alpha}{ }^{\beta} \mathcal{S}_{1 \nu \beta}+k^{\mu} u_{1 \alpha} u_{1 \nu}\left(\mathcal{S}_{1 \beta \rho} \mathcal{S}_{1}{ }^{\beta \rho}-2 u_{2}{ }^{\beta} u_{2}{ }^{\rho} \mathcal{S}_{1 \beta}{ }^{\gamma} \mathcal{S}_{1 \rho \gamma}\right)\right. \\
& \left.\quad+2 k^{\beta} u_{1 \alpha}\left(\mathcal{S}_{1 \beta \rho} \mathcal{S}_{1 \nu}{ }^{\rho}\left(u_{1}{ }^{\mu}-2 \gamma u_{2}{ }^{\mu}\right)+u_{1 \nu}\left(2 u_{2}{ }^{\mu} u_{2}{ }^{\rho} \mathcal{S}_{1 \beta}{ }^{\gamma} \mathcal{S}_{1 \rho \gamma}-\mathcal{S}_{1 \beta \rho} \mathcal{S}_{1}{ }^{\mu \rho}\right)\right)\right]
\end{aligned}
$$


so that

$$
\begin{aligned}
\delta_{E S_{1}^{2}}^{(1)} x_{1}^{\mu}\left(\tau_{1}\right)= & \frac{-i m_{2}}{16 M_{\mathrm{Pl}}^{2} m_{1}^{2}} \int_{k} \frac{\hat{\delta}\left(k \cdot u_{2}\right) k^{\alpha} k^{\nu}}{k^{2}\left(k \cdot u_{1}-i \epsilon\right)^{2}} e^{i k \cdot b} e^{i\left(k \cdot u_{1}-i \epsilon\right) \tau_{1}} \\
& \times\left[k^{\mu}\left(2 \gamma^{2}-1\right) \mathcal{S}_{1 \alpha}{ }^{\beta} \mathcal{S}_{1 \nu \beta}+k^{\mu} u_{1 \alpha} u_{1 \nu}\left(\mathcal{S}_{1 \beta \rho} \mathcal{S}_{1}{ }^{\beta \rho}-2 u_{2}{ }^{\beta} u_{2}{ }^{\rho} \mathcal{S}_{1 \beta}{ }^{\gamma} \mathcal{S}_{1 \rho \gamma}\right)\right. \\
& \left.\quad+2 k^{\beta} u_{1 \alpha}\left(\mathcal{S}_{1 \beta \rho} \mathcal{S}_{1 \nu}{ }^{\rho}\left(u_{1}{ }^{\mu}-2 \gamma u_{2}{ }^{\mu}\right)+u_{1 \nu}\left(2 u_{2}{ }^{\mu} u_{2}{ }^{\rho} \mathcal{S}_{1 \beta}{ }^{\gamma} \mathcal{S}_{1 \rho \gamma}-\mathcal{S}_{1 \beta \rho} \mathcal{S}_{1}{ }^{\mu \rho}\right)\right)\right] .
\end{aligned}
$$

Finally, we also have $\operatorname{spin}_{1}$-spin 2 corrections to the trajectories given by

$$
\begin{aligned}
\delta_{S_{1} S_{2}}^{(1)} v_{1}^{\mu}\left(\tau_{1}\right)= & \frac{1}{8 M_{\mathrm{Pl}}^{2} m_{1}} \int_{k} \frac{\hat{\delta}\left(k \cdot u_{2}\right) k^{\alpha} k^{\nu}}{k^{2}\left(k \cdot u_{1}-i \epsilon\right)} e^{i k \cdot b} e^{i\left(k \cdot u_{1}-i \epsilon\right) \tau_{1}} \\
& \times\left[k^{\mu} \mathcal{S}_{2 \nu \beta}\left(\gamma \mathcal{S}_{1 \alpha}{ }^{\beta}+u_{1}{ }^{\beta} u_{2}{ }^{\rho} \mathcal{S}_{1 \alpha \rho}\right)+k^{\beta} u_{1 \alpha}\left(u_{2}{ }^{\rho} \mathcal{S}_{1 \nu \rho} \mathcal{S}_{2}{ }^{\mu}{ }_{\beta}-u_{2}{ }^{\mu} \mathcal{S}_{1 \nu}{ }^{\rho} \mathcal{S}_{2 \beta \rho}\right)\right],
\end{aligned}
$$

and

$$
\begin{aligned}
\delta_{S_{1} S_{2}}^{(1)} x_{1}^{\mu}\left(\tau_{1}\right)= & \frac{-i}{8 M_{\mathrm{Pl}}^{2} m_{1}} \int_{k} \frac{\hat{\delta}\left(k \cdot u_{2}\right) k^{\alpha} k^{\nu}}{k^{2}\left(k \cdot u_{1}-i \epsilon\right)^{2}} e^{i k \cdot b} e^{i\left(k \cdot u_{1}-i \epsilon\right) \tau_{1}} \\
& \times\left[k^{\mu} \mathcal{S}_{2 \nu \beta}\left(\gamma \mathcal{S}_{1 \alpha}{ }^{\beta}+u_{1}{ }^{\beta} u_{2}{ }^{\rho} \mathcal{S}_{1 \alpha \rho}\right)+k^{\beta} u_{1 \alpha}\left(u_{2}{ }^{\rho} \mathcal{S}_{1 \nu \rho} \mathcal{S}_{2}{ }^{\mu}{ }_{\beta}-u_{2}{ }^{\mu} \mathcal{S}_{1 \nu}{ }^{\rho} \mathcal{S}_{2 \beta \rho}\right)\right] .
\end{aligned}
$$

\section{A.1.2 Spin}

Integrating the spin equation in (2.9) we find (with the notation $k^{[\alpha} q^{\beta]}=k^{\alpha} q^{\beta}-k^{\beta} q^{\alpha}$ )

$$
\begin{aligned}
\delta_{S_{1}}^{(1)} S_{1}^{\alpha \beta}\left(\tau_{1}\right)=-\frac{m_{2}}{8 M_{\mathrm{Pl}}^{2}} \int_{k} \frac{\hat{\delta}\left(k \cdot u_{2}\right)}{k^{2}\left(k \cdot u_{1}-i \epsilon\right)} e^{i k \cdot b} e^{i\left(k \cdot u_{1}-i \epsilon\right) \tau_{1}} \\
\quad \times\left(2 \gamma u_{2}{ }^{\rho} k^{[\alpha} \mathcal{S}_{1}^{\beta]}{ }_{\rho}^{\beta}-k^{\rho} \mathcal{S}_{1}{ }_{\rho}{ }_{\rho}^{\alpha}\left(u_{1}-2 \gamma u_{2}\right)^{\beta]}\right),
\end{aligned}
$$

for the linear term, whereas at quadratic order

$$
\begin{aligned}
& \delta_{S_{1} S_{2}}^{(1)} S_{1}^{\alpha \beta}\left(\tau_{1}\right)=-\frac{i}{8 M_{\mathrm{Pl}}^{2}} \int_{k} \frac{\hat{\delta}\left(k \cdot u_{2}\right) k^{\rho}}{k^{2}\left(k \cdot u_{1}-i \epsilon\right)} e^{i k \cdot b} e^{i\left(k \cdot u_{1}-i \epsilon\right) \tau_{1}} \\
& \times\left(k^{\sigma} u_{1}{ }^{\nu} u_{2}{ }^{[\alpha} \mathcal{S}_{1}^{\beta]}{ }_{\rho} \mathcal{S}_{2 \sigma \nu}+\gamma k^{\sigma} \mathcal{S}_{1}{ }_{\rho}^{\alpha} \mathcal{S}_{2}{ }^{\beta]}{ }_{\sigma}\right. \\
& \left.-\mathcal{S}_{2 \rho \sigma}\left(\gamma k^{[\alpha} \mathcal{S}_{1}^{\beta] \sigma}+u_{1}^{\sigma} u_{2}^{\nu} k^{[\alpha} \mathcal{S}_{1}^{\beta}{ }_{\nu}\right)\right) \\
& \delta_{E S_{1}^{2}}^{(1)} S_{1}^{\alpha \beta}\left(\tau_{1}\right)=\frac{-i m_{2}}{8 M_{\mathrm{Pl}}^{2} m_{1}} \mathcal{S}_{1 \rho \sigma} \int_{k} \frac{\hat{\delta}\left(k \cdot u_{2}\right)}{k^{2}\left(k \cdot u_{1}-i \epsilon\right)} e^{i k \cdot b} e^{i\left(k \cdot u_{1}-i \epsilon\right) \tau_{1}} \\
& \times\left(\left(k \cdot u_{1}\right)^{2} 2 u_{2}{ }^{\sigma} u_{2}{ }^{[\alpha} \mathcal{S}_{1}^{\beta] \rho}+k^{\rho}\left(k \cdot u_{1}\right) \mathcal{S}_{1}{ }^{[\alpha \sigma}\left(u_{1}-2 \gamma u_{2}\right)^{\beta]}\right. \\
& \left.-k^{[\alpha} \mathcal{S}_{1}^{\beta] \sigma}\left(\left(2 \gamma^{2}-1\right) k^{\rho}-2 \gamma u_{2}^{\rho}\left(k \cdot u_{1}\right)\right)\right) \\
& \delta_{R S_{1} S_{1}}^{(1)} S_{1}^{\alpha \beta}\left(\tau_{1}\right)=\frac{i m_{2}}{8 M_{\mathrm{Pl}}^{2} m_{1}} \int_{k} \frac{\hat{\delta}\left(k \cdot u_{2}\right) k^{\rho}}{k^{2}\left(k \cdot u_{1}-i \epsilon\right)} e^{i k \cdot b} e^{i\left(k \cdot u_{1}-i \epsilon\right) \tau_{1}}\left(2 \gamma k^{\sigma} u_{2}{ }^{\nu} \mathcal{S}_{1 \sigma \nu} u_{1}{ }^{[\alpha} \mathcal{S}_{1}{ }^{\beta]}{ }_{\rho}\right. \\
& \left.+\left(k \cdot u_{1}\right)\left(u_{1}^{[\alpha} \mathcal{S}_{1}^{\beta] \sigma} \mathcal{S}_{1 \rho \sigma}-2 u_{2}^{\nu} u_{2}^{\sigma} \mathcal{S}_{1 \rho \nu} u_{1}^{[\alpha} \mathcal{S}_{1}^{\beta]}\right)\right) \text {. }
\end{aligned}
$$




\section{A.2 Scattering data}

The value of the $D_{i}$ coefficients for the total momentum and spin impulses are given by:

$$
\begin{aligned}
& D_{1}=\frac{\pi \gamma\left(5 \gamma^{2}-3\right)(\delta+7)}{8\left(\gamma^{2}-1\right)^{3 / 2}} \\
& D_{2}=\frac{1}{\gamma^{2}-1}\left[\left(8 \gamma^{3}+4 \gamma^{2}-4 \gamma-1\right)+\left(8 \gamma^{3}-4 \gamma^{2}-4 \gamma+1\right) \delta\right] \\
& D_{3}=\frac{\gamma}{\left(\gamma^{2}-1\right)^{2}}\left[(2 \gamma+1)\left(8 \gamma^{2}+2 \gamma-5\right)+(2 \gamma-1)\left(8 \gamma^{2}-2 \gamma-5\right) \delta\right] \\
& D_{4}=\frac{1}{\left(\gamma^{2}-1\right)^{2}}\left[\left(-8 \gamma^{4}-16 \gamma^{3}+8 \gamma+1\right)+\left(8 \gamma^{4}-16 \gamma^{3}+8 \gamma-1\right) \delta\right] \\
& D_{5}=\frac{3 \pi}{128 \sqrt{\gamma^{2}-1}}\left(35 \gamma^{2}(\delta+1)+9 \delta+1\right) \\
& +\frac{3 \pi C_{E S}^{(1)}}{128\left(\gamma^{2}-1\right)^{3 / 2}}\left(5 \gamma^{4}(7 \delta+55)-58 \gamma^{2}(\delta+5)+23 \delta+47\right) \\
& D_{6}=-\frac{2 \gamma}{\gamma^{2}-1}[(2 \gamma+1)+(2 \gamma-1) \delta] \\
& -\frac{\left(2 \gamma^{2}-1\right)}{\left(\gamma^{2}-1\right)^{2}} C_{E S}^{(1)}\left[\left(2 \gamma^{2}+\gamma-1\right)+\left(2 \gamma^{2}-\gamma-1\right) \delta\right] \\
& D_{7}=\frac{\left(2 \gamma^{2}-1\right)^{2}}{\left(\gamma^{2}-1\right)^{2}} C_{E S}^{(1)}[(\gamma+1)+(\gamma-1) \delta], \quad D_{8}=D_{7}(\delta \leftrightarrow-\delta) \\
& D_{9}=\frac{3 \pi}{128 \sqrt{\gamma^{2}-1}}\left(5 \gamma^{2}(7 \delta+31)+\delta-15\right) \\
& +\frac{3 \pi C_{E S}^{(1)}}{128\left(\gamma^{2}-1\right)^{3 / 2}}\left(5 \gamma^{4}(7 \delta+31)-2 \gamma^{2}(25 \delta+77)+15 \delta+31\right) \\
& D_{10}=\frac{3 \pi}{64\left(\gamma^{2}-1\right)^{3 / 2}}\left(5 \gamma^{2}(7 \delta+19)-3 \delta+1\right) \\
& +\frac{3 \pi C_{E S}^{(1)}}{64\left(\gamma^{2}-1\right)^{5 / 2}}\left(5 \gamma^{4}(7 \delta+19)-2 \gamma^{2}(23 \delta+43)+11 \delta+23\right) \\
& D_{11}=\frac{4 \gamma^{2}}{\left(\gamma^{2}-1\right)^{2}}[(3 \gamma+2)+(3 \gamma-2) \delta] \\
& +\frac{\left(2 \gamma^{2}-1\right)}{\left(\gamma^{2}-1\right)^{3}} C_{E S}^{(1)}\left[(\gamma+1)\left(6 \gamma^{2}-2 \gamma-1\right)+(\gamma-1)\left(6 \gamma^{2}+2 \gamma-1\right) \delta\right] \\
& D_{12}=\frac{4 \gamma}{\left(\gamma^{2}-1\right)^{2}}\left[\left(-\gamma^{2}-3 \gamma-1\right)+\left(\gamma^{2}-3 \gamma+1\right) \delta\right] \\
& +\frac{\left(2 \gamma^{2}-1\right)}{\left(\gamma^{2}-1\right)^{3}} C_{E S}^{(1)}\left[(\gamma-1)\left(2 \gamma^{2}-4 \gamma-3\right)+(\gamma-1)\left(2 \gamma^{2}-4 \gamma-3\right) \delta\right] \\
& D_{13}=\frac{4 \gamma^{2}}{\gamma^{2}-1}[(\gamma+1)+(\gamma-1) \delta]+D_{7}, \quad D_{14}=D_{13}(\delta \leftrightarrow-\delta) \\
& D_{15}=\frac{3 \pi\left(20 \gamma^{4}-21 \gamma^{2}+3\right)}{4\left(\gamma^{2}-1\right)^{3 / 2}}
\end{aligned}
$$




$$
\begin{aligned}
& D_{16}=\frac{1}{\left(\gamma^{2}-1\right)^{2}}\left[(\gamma+1)\left(8 \gamma^{3}-4 \gamma^{2}-4 \gamma+1\right)-(\gamma-1)\left(8 \gamma^{3}+4 \gamma^{2}-4 \gamma-1\right) \delta\right] \\
& D_{17}=2 \frac{\left(2 \gamma^{2}-1\right)^{2}}{\left(\gamma^{2}-1\right)^{2}}[(\gamma+1)+(\gamma-1) \delta] \\
& D_{18}=\frac{3 \pi \gamma^{3}\left(4-5 \gamma^{2}\right)}{\left(\gamma^{2}-1\right)^{5 / 2}} \\
& D_{19}=-\frac{2}{\left(\gamma^{2}-1\right)^{3}}\left[(\gamma+1)\left(8 \gamma^{5}+8 \gamma^{4}-4 \gamma^{3}-8 \gamma^{2}-2 \gamma+1\right)\right. \\
& \left.+(\gamma-1)\left(8 \gamma^{5}-8 \gamma^{4}-4 \gamma^{3}+8 \gamma^{2}-2 \gamma-1\right) \delta\right] \\
& D_{20}=\frac{2}{\left(\gamma^{2}-1\right)^{2}}\left[(\gamma+1)\left(8 \gamma^{4}-8 \gamma^{2}+1\right)+(\gamma-1)\left(8 \gamma^{4}-8 \gamma^{2}+1\right) \delta\right] \\
& D_{21}=-\frac{\left(8 \gamma^{4}-8 \gamma^{2}+1\right)(\delta-1)}{2\left(\gamma^{2}-1\right)} \\
& D_{22}=\frac{3 \pi\left(5 \gamma^{2}-1\right)}{4 \sqrt{\gamma^{2}-1}} \\
& D_{23}=4\left(1-2 \gamma^{2}\right)^{2} D_{25} \\
& D_{24}=\frac{\left(2 \gamma^{2}-1\right)\left(2 \gamma^{2}-4 \gamma+1\right)}{(\gamma-1)^{2}(\gamma+1)}-\frac{\left(2 \gamma^{2}-1\right)\left(2 \gamma^{2}+4 \gamma+1\right) \delta}{(\gamma-1)(\gamma+1)^{2}} \\
& D_{25}=\frac{\delta-1}{2\left(\gamma^{2}-1\right)^{2}} \\
& D_{26}=-\frac{2 \gamma\left(2 \gamma^{2}-1\right)(\delta-1)\left(C_{E S^{2}}^{(1)}+1\right)}{\gamma^{2}-1}=\frac{\left(C_{E S^{2}}^{(1)}+1\right)}{2} D_{28} \\
& D_{27}=-\frac{4 \gamma(\delta-1)}{\gamma^{2}-1} \\
& D_{28}=\left(2 \gamma^{2}-1\right) D_{27} \\
& D_{29}=-\frac{2\left(1-2 \gamma^{2}\right)^{2}(\delta-1) C_{E S^{2}}^{(1)}}{\gamma^{2}-1} \\
& D_{30}=-\frac{8 \gamma\left(2 \gamma^{2}-1\right)\left(C_{E S^{2}}^{(1)}-3\right) \nu}{\left(\gamma^{2}-1\right)(\delta+1)} \\
& D_{31}=\frac{8 \gamma\left(2 \gamma^{2}-1\right)\left(C_{E S^{2}}^{(1)}-1\right) \nu}{\left(\gamma^{2}-1\right)^{2}(\delta+1)} \\
& D_{32}=-\frac{8 \gamma}{\left(\gamma^{2}-1\right)(\delta+1)}\left(8 \gamma^{2} \nu+\gamma(\delta-2 \nu+1)-3 \nu\right) \\
& +\frac{2\left(2 \gamma^{2}-1\right) C_{E S^{2}}^{(1)}}{\left(\gamma^{2}-1\right)^{2}(\delta+1)}\left(8 \gamma^{3} \nu+\gamma^{2}(2 \delta-4 \nu+2)-6 \gamma \nu-\delta+2 \nu-1\right) \\
& D_{33}=-\frac{4 \gamma}{\left(\gamma^{2}-1\right)^{2}(\delta+1)}\left(8 \gamma^{4} \nu+\gamma^{3}(12 \nu-6(\delta+1))-22 \gamma^{2} \nu+\gamma(2 \delta-4 \nu+2)+7 \nu\right) \\
& +\frac{2\left(2 \gamma^{2}-1\right) C_{E S^{2}}^{(1)}}{\left(\gamma^{2}-1\right)^{2}(\delta+1)}\left(8 \gamma^{3} \nu+\gamma^{2}(-2 \delta+4 \nu-2)-10 \gamma \nu+\delta-2 \nu+1\right)
\end{aligned}
$$




$$
\begin{aligned}
& D_{34}=\frac{4}{\left(\gamma^{2}-1\right)^{2}(\delta+1)}\left(-8 \gamma^{4} \nu+\gamma^{3}(-8 \delta+16 \nu-8)+\gamma(4 \delta-8 \nu+4)+\nu\right) \\
& D_{35}=\frac{\pi \gamma\left(5 \gamma^{2}-3\right)(7-\delta)}{8\left(\gamma^{2}-1\right)^{3 / 2}=\frac{7-\delta}{\delta+7} D_{1}} \\
& D_{36}=\frac{\pi \gamma^{2}\left(3-5 \gamma^{2}\right)}{4\left(\gamma^{2}-1\right)^{3 / 2}=-\frac{2 \gamma}{\delta+7} D_{1}} \\
& D_{37}=-\frac{D_{22}}{\gamma^{2}-1} \\
& D_{38}=\frac{2\left(2 \gamma^{2}+1\right)(\delta-1)}{\gamma^{2}-1} \\
& D_{39}=\frac{\gamma(1-\delta)+\delta+1}{\left(\gamma^{2}-1\right)^{2}} \\
& D_{40}=\frac{\left(8 \gamma^{3}-4 \gamma^{2}-4 \gamma+1\right) \delta}{\gamma^{2}-1}+\frac{\left(-8 \gamma^{3}-4 \gamma^{2}+4 \gamma+1\right)}{\gamma^{2}-1} \\
& D_{41}=-\frac{\gamma(2 \gamma+1)\left(8 \gamma^{2}+2 \gamma-5\right)}{\left(\gamma^{2}-1\right)^{2}}+\frac{\gamma(2 \gamma-1)\left(8 \gamma^{2}-2 \gamma-5\right) \delta}{\left(\gamma^{2}-1\right)^{2}} \\
& D_{42}=\frac{\gamma\left(-8 \gamma^{4}+12 \gamma^{3}-4 \gamma^{2}-5 \gamma+4\right) \delta}{\left(\gamma^{2}-1\right)^{2}}+\frac{\gamma\left(8 \gamma^{4}+12 \gamma^{3}+4 \gamma^{2}-5 \gamma-4\right)}{\left(\gamma^{2}-1\right)^{2}} \\
& D_{43}=\frac{\pi \gamma\left(5 \gamma^{4}+2 \gamma^{2}-3\right)}{4\left(\gamma^{2}-1\right)^{5 / 2}}=\frac{\gamma^{2}+1}{\gamma\left(1-\gamma^{2}\right)} D_{36} \\
&
\end{aligned}
$$

\section{B One-loop integration}

At 2PM order, all expressions for momentum and spin impulses from Feynman diagrams contain one-loop tensor integrals of the form:

$$
I_{\left(a_{1}, a_{2}, a_{3}\right)}^{\mu_{1} \cdots \mu_{m}}=\int_{k} \frac{\hat{\delta}\left(k \cdot u_{j}\right) k^{\mu_{1}} \cdots k^{\mu_{m}}}{\left[k^{2}\right]^{a_{1}}\left[(k-q)^{2}\right]^{a_{2}}\left( \pm k \cdot u_{j}-i \epsilon\right)^{a_{3}}},
$$

with $q \cdot u_{1}=q \cdot u_{2}=0$ and we use the convention $\{\mathfrak{1}=2, \mathfrak{z}=1\}$, introduced in [83]. The linear propagators appear due to the iterations where we input the trajectories shown in appendix A in the tree-level Routhian/action. In non-spinning cases [82-84], all integrals have at most one Lorentz index in the numerator. The situation changes when spin is included, and we find various tensor integrals of rank $m \in\{0,1,2,3\}$. Following the standard method first proposed by Passarino and Veltman [157], we reduce all the one-loop tensor integral to a linear combination of scalar integrals. The idea is simple, Lorentz covariance implies that the tensor structure in the final results can be constructed in terms of only the external data $\left\{q^{\mu}, u_{1}^{\mu}, u_{2}^{\mu}\right\}$ and the metric tensor $g^{\mu \nu}$. Let us consider for example the integral, which we encountered in the non-spinning case [82],

$$
I_{\left(a_{1}, a_{2}, a_{3}\right)}^{\mu}=\int_{k} \frac{\hat{\delta}\left(k \cdot u_{2}\right) k^{\mu}}{\left[k^{2}\right]^{a_{1}}\left[(k-q)^{2}\right]^{a_{2}}\left( \pm k \cdot u_{1}-i \epsilon\right)^{a_{3}}},
$$

with $a_{3}>0$. Hence, the tensor decomposition yields

$$
I_{\left(a_{1}, a_{2}, a_{3}\right)}^{\mu}=q^{\mu} I_{q}+u_{1}^{\mu} I_{u_{1}}+u_{2}^{\mu} I_{u_{2}},
$$


with the scalar integrals $I_{q}, I_{u_{1}}$ and $I_{u_{2}}$ often denoted as 'form factors' in the literature. We can now solve the form factors by performing Lorentz contractions on both sides, with $q, u_{1}$ and $u_{2}$. In particular, by contracting with $u_{2}$ we immediately find $I_{u_{2}}=-\gamma I_{u_{1}}$ (reflecting the fact that the integral in (B.2) must be perpendicular to $u_{2}$ ). As a result, we must compute only the scalar integrals

$$
\begin{aligned}
I_{q} & =\frac{1}{q^{2}} \int_{k} \frac{\hat{\delta}\left(k \cdot u_{2}\right) k \cdot q}{\left[k^{2}\right]^{a_{1}}\left[(k-q)^{2}\right]^{a_{2}}\left( \pm k \cdot u_{1}-i \epsilon\right)^{a_{3}}}, \\
I_{u_{1}} & =-\frac{1}{\gamma^{2}-1} \int_{k} \frac{\hat{\delta}\left(k \cdot u_{2}\right)}{\left[k^{2}\right]^{a_{1}}\left[(k-q)^{2}\right]^{a_{2}}\left( \pm k \cdot u_{1}-i \epsilon\right)^{a_{3}-1}} .
\end{aligned}
$$

At the end of the day, going to the rest frame of particle 2 to resolve the delta function, all of these integrals belong to the following family: ${ }^{11}$

$$
\int_{k} \frac{1}{\left(\boldsymbol{k}^{2}\right)^{a_{1}}\left[(\boldsymbol{k}-\boldsymbol{q})^{2}\right]^{a_{2}}( \pm \boldsymbol{k} \cdot \boldsymbol{u}-i \epsilon)^{a_{3}}},
$$

with $\boldsymbol{q} \cdot \boldsymbol{u}=0$ and $\boldsymbol{u}^{2}=\gamma^{2}-1$, in $D=3-2 \epsilon$ dimensions. While analytical expressions for any $\left\{a_{1}, a_{2}, a_{3}\right\}$ are known, e.g. [158], it is often convenient to use integration-by-parts (IBP) relations to reduce the integrals in (B.6) to a combination of the following masters

$$
\begin{aligned}
\int \frac{d^{D} k}{\pi^{D / 2}} \frac{1}{\boldsymbol{k}^{2}(\boldsymbol{k}-\boldsymbol{q})^{2}} & =\frac{1}{\left(\boldsymbol{q}^{2}\right)^{2-D / 2}} \frac{\Gamma(2-D / 2) \Gamma^{2}(D / 2-1)}{\Gamma(D-2)} \\
\int \frac{d^{D} k}{\pi^{D / 2}} \frac{1}{\boldsymbol{k}^{2}(\boldsymbol{k}-\boldsymbol{q})^{2}( \pm \boldsymbol{k} \cdot \boldsymbol{u}-i \epsilon)} & =i \frac{\sqrt{\pi}}{\left(\boldsymbol{q}^{2}\right)^{(5-D) / 2} \sqrt{\gamma^{2}-1}} \frac{\Gamma((5-D) / 2) \Gamma^{2}((D-3) / 2)}{\sqrt{\gamma^{2}-1} \Gamma(D-3)}
\end{aligned}
$$

with the factor of $\pi^{D / 2}$ introduced to comply with the present literature. Similar considerations apply to higher-ranked tensor decompositions.

Finally, we must perform the Fourier transform to impact parameter space, which can be written in terms of derivatives w.r.t. to $b^{\mu}$,

$$
\int_{q} \frac{e^{i q \cdot b} \hat{\delta}\left(q \cdot u_{1}\right) \hat{\delta}\left(q \cdot u_{2}\right) q^{\mu_{1}} \cdots q^{\mu_{m}}}{\left(-q^{2}\right)^{n}}=\left(-i \partial_{b}^{\mu_{1}}\right) \cdots\left(-i \partial_{b}^{\mu_{m}}\right) \int_{q} \frac{e^{i q \cdot b} \hat{\delta}\left(q \cdot u_{1}\right) \hat{\delta}\left(q \cdot u_{2}\right)}{\left(-q^{2}\right)^{n}} .
$$

We first notice that the results must lie in the plane orthogonal to both $u_{1}$ and $u_{2}$. We can then construct a projected metric $[97,135]$

$$
\frac{\partial}{\partial b_{\mu}} b^{\nu}=\Pi^{\mu \nu}=\eta^{\mu \nu}+\frac{u_{1}^{\mu}\left(u_{1}^{\nu}-\gamma u_{2}^{\nu}\right)+u_{2}^{\mu}\left(u_{2}^{\nu}-\gamma u_{1}^{\nu}\right)}{\gamma^{2}-1},
$$

which we can use to reduce into scalar integrals. Using (B.9), together with (B.10), and the master integral

$$
\int_{q} \frac{e^{i q \cdot b} \hat{\delta}\left(q \cdot u_{1}\right) \hat{\delta}\left(q \cdot u_{2}\right)}{\left(-q^{2}\right)^{n}}=\frac{4^{-n} \pi^{(2-D) / 2}}{\sqrt{\gamma^{2}-1}|b|^{D-2-2 n}} \frac{\Gamma\left(\frac{D-2}{2}-n\right)}{\Gamma(n)},
$$

\footnotetext{
${ }^{11}$ Alternatively, as shown in [82], we can also perform the $k^{0}$ integral in the rest frame of particle 1 and pick up the (conservative) pole from the linear propagator only.
} 
it is straightforward to generate the Fourier integrals of any rank, e.g.

$$
\begin{aligned}
& \int_{q} \frac{e^{i q \cdot b} \hat{\delta}\left(q \cdot u_{1}\right) \hat{\delta}\left(q \cdot u_{2}\right) q^{\mu} q^{\nu}}{\left(-q^{2}\right)^{n}} \\
& \quad=-\frac{2^{1-2 n} \pi^{(2-D) / 2}}{\sqrt{\gamma^{2}-1}|b|^{D+2-2 n}} \frac{\Gamma(D / 2-n)}{\Gamma(n)}\left((D-2 n) b^{\mu} b^{\nu}+|b|^{2} \Pi^{\mu \nu}\right) .
\end{aligned}
$$

Open Access. This article is distributed under the terms of the Creative Commons Attribution License (CC-BY 4.0), which permits any use, distribution and reproduction in any medium, provided the original author(s) and source are credited.

\section{References}

[1] LIGO ScientifiC and Virgo collaborations, Open data from the first and second observing runs of Advanced LIGO and Advanced Virgo, SoftwareX 13 (2021) 100658 [arXiv: 1912.11716] [INSPIRE].

[2] A. Buonanno and B.S. Sathyaprakash, Sources of Gravitational Waves: Theory and Observations, arXiv: 1410.7832 [INSPIRE].

[3] R.A. Porto, The Tune of Love and the Nature(ness) of Spacetime, Fortsch. Phys. 64 (2016) 723 [arXiv: 1606.08895$]$ [INSPIRE].

[4] R.A. Porto, The Music of the Spheres: The Dawn of Gravitational Wave Science, arXiv: 1703.06440 [INSPIRE].

[5] S. Vitale, R. Lynch, J. Veitch, V. Raymond and R. Sturani, Measuring the spin of black holes in binary systems using gravitational waves, Phys. Rev. Lett. 112 (2014) 251101 [arXiv: 1403.0129] [INSPIRE].

[6] B. Zackay, T. Venumadhav, L. Dai, J. Roulet and M. Zaldarriaga, Highly spinning and aligned binary black hole merger in the Advanced LIGO first observing run, Phys. Rev. D 100 (2019) 023007 [arXiv: 1902.10331] [INSPIRE].

[7] A. Arvanitaki and S. Dubovsky, Exploring the String Axiverse with Precision Black Hole Physics, Phys. Rev. D 83 (2011) 044026 [arXiv: 1004.3558] [InSPIRE].

[8] A. Arvanitaki, M. Baryakhtar, S. Dimopoulos, S. Dubovsky and R. Lasenby, Black Hole Mergers and the QCD Axion at Advanced LIGO, Phys. Rev. D 95 (2017) 043001 [arXiv: 1604.03958] [INSPIRE].

[9] R. Brito et al., Stochastic and resolvable gravitational waves from ultralight bosons, Phys. Rev. Lett. 119 (2017) 131101 [arXiv:1706.05097] [INSPIRE].

[10] K.K.Y. Ng, M. Isi, C.-J. Haster and S. Vitale, Multiband gravitational-wave searches for ultralight bosons, Phys. Rev. D 102 (2020) 083020 [arXiv:2007.12793] [InSPIRE].

[11] K.K.Y. Ng, O.A. Hannuksela, S. Vitale and T.G.F. Li, Searching for ultralight bosons within spin measurements of a population of binary black hole mergers, Phys. Rev. D 103 (2021) 063010 [arXiv: 1908.02312] [inSPIRE].

[12] K.K.Y. Ng, S. Vitale, O.A. Hannuksela and T.G.F. Li, Constraints on Ultralight Scalar Bosons within Black Hole Spin Measurements from the LIGO-Virgo GWTC-2, Phys. Rev. Lett. 126 (2021) 151102 [arXiv: 2011.06010] [INSPIRE]. 
[13] D. Baumann, H.S. Chia and R.A. Porto, Probing Ultralight Bosons with Binary Black Holes, Phys. Rev. D 99 (2019) 044001 [arXiv:1804.03208] [INSPIRE].

[14] D. Baumann, H.S. Chia, R.A. Porto and J. Stout, Gravitational Collider Physics, Phys. Rev. D 101 (2020) 083019 [arXiv: 1912.04932] [InSPIRE].

[15] R.P. Kerr, Rotating black holes and the Kerr metric, AIP Conf. Proc. 1059 (2008) 9 [INSPIRE].

[16] M. Mathisson, Neue mechanik materieller systemes, Acta Phys. Polon. 6 (1937) 163 [INSPIRE].

[17] A. Papapetrou, Spinning test particles in general relativity. 1, Proc. Roy. Soc. Lond. A 209 (1951) 248 [INSPIRE].

[18] E. Corinaldesi and A. Papapetrou, Spinning test particles in general relativity. 2, Proc. Roy. Soc. Lond. A 209 (1951) 259 [InSPIRE].

[19] W.G. Dixon, Dynamics of extended bodies in general relativity. I. Momentum and angular momentum, Proc. Roy. Soc. Lond. A 314 (1970) 499 [inSPIRE].

[20] B.M. Barker and R.F. O'Connell, Gravitational Two-Body Problem with Arbitrary Masses, Spins, and Quadrupole Moments, Phys. Rev. D 12 (1975) 329 [INSPIRE].

[21] I. Bailey and W. Israel, Lagrangian Dynamics of Spinning Particles and Polarized Media in General Relativity, Commun. Math. Phys. 42 (1975) 65 [InSPIRE].

[22] L.E. Kidder, C.M. Will and A.G. Wiseman, Spin effects in the inspiral of coalescing compact binaries, Phys. Rev. D 47 (1993) R4183 [gr-qc/9211025] [inSPIRE].

[23] L.E. Kidder, C.M. Will and A.G. Wiseman, Coalescing binary systems of compact objects to (post)**(5/2)-Newtonian order. 3. Transition from inspiral to plunge, Phys. Rev. D 47 (1993) 3281 [INSPIRE].

[24] L.E. Kidder, Coalescing binary systems of compact objects to postNewtonian 5/2 order. 5. Spin effects, Phys. Rev. D 52 (1995) 821 [gr-qc/9506022] [INSPIRE].

[25] B.J. Owen, H. Tagoshi and A. Ohashi, Nonprecessional spin orbit effects on gravitational waves from inspiraling compact binaries to second postNewtonian order, Phys. Rev. D $\mathbf{5 7}$ (1998) 6168 [gr-qc/9710134] [INSPIRE].

[26] H. Tagoshi, A. Ohashi and B.J. Owen, Gravitational field and equations of motion of spinning compact binaries to 2.5 postNewtonian order, Phys. Rev. D 63 (2001) 044006 [gr-qc/0010014] [INSPIRE].

[27] G. Faye, L. Blanchet and A. Buonanno, Higher-order spin effects in the dynamics of compact binaries. I. Equations of motion, Phys. Rev. D 74 (2006) 104033 [gr-qc/0605139] [INSPIRE].

[28] L. Blanchet, A. Buonanno and G. Faye, Higher-order spin effects in the dynamics of compact binaries. II. Radiation field, Phys. Rev. D $\mathbf{7 4}$ (2006) 104034 [Erratum ibid. $\mathbf{7 5}$ (2007) 049903] [Erratum ibid. 81 (2010) 089901] [gr-qc/0605140] [INSPIRE].

[29] I. Hinder, S. Ossokine, H.P. Pfeiffer and A. Buonanno, Gravitational waveforms for high spin and high mass-ratio binary black holes: A synergistic use of numerical-relativity codes, Phys. Rev. D 99 (2019) 061501 [arXiv:1810.10585] [INSPIRE].

[30] W.D. Goldberger and I.Z. Rothstein, An effective field theory of gravity for extended objects, Phys. Rev. D 73 (2006) 104029 [hep-th/0409156] [INSPIRE]. 
[31] R.A. Porto, Post-Newtonian corrections to the motion of spinning bodies in NRGR, Phys. Rev. D 73 (2006) 104031 [gr-qc/0511061] [INSPIRE].

[32] W.D. Goldberger, Les Houches lectures on effective field theories and gravitational radiation, in Les Houches Summer School - Session 86: Particle Physics and Cosmology: The Fabric of Spacetime, (2007) [hep-ph/0701129] [INSPIRE].

[33] S. Foffa and R. Sturani, Effective field theory methods to model compact binaries, Class. Quant. Grav. 31 (2014) 043001 [arXiv: 1309.3474] [INSPIRE].

[34] R.A. Porto, The effective field theorist's approach to gravitational dynamics, Phys. Rept. 633 (2016) 1 [arXiv: 1601.04914] [INSPIRE].

[35] R.A. Porto and I.Z. Rothstein, The hyperfine Einstein-Infeld-Hoffmann potential, Phys. Rev. Lett. 97 (2006) 021101 [gr-qc/0604099] [INSPIRE].

[36] R.A. Porto, New results at 3PN via an effective field theory of gravity, in 11th Marcel Grossmann Meeting on General Relativity, (2007), DOI [gr-qc/0701106] [INSPIRE].

[37] R.A. Porto and I.Z. Rothstein, Spin(1)Spin(2) Effects in the Motion of Inspiralling Compact Binaries at Third Order in the Post-Newtonian Expansion, Phys. Rev. D 78 (2008) 044012 [Erratum ibid. 81 (2010) 029904] [arXiv:0802.0720] [INSPIRE].

[38] R.A. Porto and I.Z. Rothstein, Next to Leading Order Spin(1)Spin(1) Effects in the Motion of Inspiralling Compact Binaries, Phys. Rev. D 78 (2008) 044013 [Erratum ibid. 81 (2010) 029905] [arXiv: 0804.0260] [INSPIRE].

[39] J. Steinhoff, S. Hergt and G. Schaefer, On the next-to-leading order gravitational spin(1)-spin(2) dynamics, Phys. Rev. D 77 (2008) 081501 [arXiv:0712.1716] [INSPIRE].

[40] J. Steinhoff, S. Hergt and G. Schaefer, Spin-squared Hamiltonian of next-to-leading order gravitational interaction, Phys. Rev. D 78 (2008) 101503 [arXiv:0809.2200] [INSPIRE].

[41] R.A. Porto, Next to leading order spin-orbit effects in the motion of inspiralling compact binaries, Class. Quant. Grav. 27 (2010) 205001 [arXiv: 1005. 5730] [INSPIRE].

[42] M. Levi and J. Steinhoff, Next-to-next-to-leading order gravitational spin-orbit coupling via the effective field theory for spinning objects in the post-Newtonian scheme, JCAP 01 (2016) 011 [arXiv: 1506.05056] [INSPIRE].

[43] M. Levi and J. Steinhoff, Complete conservative dynamics for inspiralling compact binaries with spins at fourth post-Newtonian order, arXiv:1607.04252 [INSPIRE].

[44] M. Levi, A.J. Mcleod and M. Von Hippel, NNNLO gravitational quadratic-in-spin interactions at the quartic order in $G$, arXiv:2003.07890 [INSPIRE].

[45] M. Levi, A.J. Mcleod and M. Von Hippel, $N^{3} L O$ gravitational spin-orbit coupling at order $G^{4}$, arXiv:2003.02827 [INSPIRE].

[46] A. Antonelli, C. Kavanagh, M. Khalil, J. Steinhoff and J. Vines, Gravitational spin-orbit coupling through third-subleading post-Newtonian order: from first-order self-force to arbitrary mass ratios, Phys. Rev. Lett. 125 (2020) 011103 [arXiv:2003.11391] [InSPIRE].

[47] A. Antonelli, C. Kavanagh, M. Khalil, J. Steinhoff and J. Vines, Gravitational spin-orbit and aligned spin $_{1}-$ spin $_{2}$ couplings through third-subleading post-Newtonian orders, Phys. Rev. D 102 (2020) 124024 [arXiv:2010.02018] [INSPIRE].

[48] R.A. Porto, A. Ross and I.Z. Rothstein, Spin induced multipole moments for the gravitational wave flux from binary inspirals to third Post-Newtonian order, JCAP 03 (2011) 009 [arXiv: 1007.1312] [INSPIRE]. 
[49] R.A. Porto, A. Ross and I.Z. Rothstein, Spin induced multipole moments for the gravitational wave amplitude from binary inspirals to 2.5 Post-Newtonian order, JCAP 09 (2012) 028 [arXiv: 1203.2962] [INSPIRE].

[50] A. Bohé, G. Faye, S. Marsat and E.K. Porter, Quadratic-in-spin effects in the orbital dynamics and gravitational-wave energy flux of compact binaries at the 3PN order, Class. Quant. Grav. 32 (2015) 195010 [arXiv:1501.01529] [INSPIRE].

[51] N.T. Maia, C.R. Galley, A.K. Leibovich and R.A. Porto, Radiation reaction for spinning bodies in effective field theory I: Spin-orbit effects, Phys. Rev. D 96 (2017) 084064 [arXiv: 1705.07934] [INSPIRE].

[52] N.T. Maia, C.R. Galley, A.K. Leibovich and R.A. Porto, Radiation reaction for spinning bodies in effective field theory II: Spin-spin effects, Phys. Rev. D 96 (2017) 084065 [arXiv: 1705. 07938] [INSPIRE].

[53] Z. Yang and A.K. Leibovich, Analytic Solutions to Compact Binary Inspirals With Leading Order Spin-Orbit Contribution Using The Dynamical Renormalization Group, Phys. Rev. D 100 (2019) 084021 [arXiv: 1908.05688] [INSPIRE].

[54] B.A. Pardo and N.T. Maia, Next-to-leading order spin-orbit effects in the equations of motion, energy loss and phase evolution of binaries of compact bodies in the effective field theory approach, Phys. Rev. D 102 (2020) 124020 [arXiv:2009.05628] [INSPIRE].

[55] G. Cho, B. Pardo and R.A. Porto, Gravitational radiation from inspiralling compact objects: Spin-spin effects completed at the next-to-leading post-Newtonian order, arXiv:2103.14612 [INSPIRE].

[56] A. Bohé, S. Marsat and L. Blanchet, Next-to-next-to-leading order spin-orbit effects in the gravitational wave flux and orbital phasing of compact binaries, Class. Quant. Grav. 30 (2013) 135009 [arXiv:1303.7412] [INSPIRE].

[57] W.D. Goldberger and A. Ross, Gravitational radiative corrections from effective field theory, Phys. Rev. D 81 (2010) 124015 [arXiv:0912.4254] [InSPIRE].

[58] A.K. Leibovich, N.T. Maia, I.Z. Rothstein and Z. Yang, Second post-Newtonian order radiative dynamics of inspiralling compact binaries in the Effective Field Theory approach, Phys. Rev. D 101 (2020) 084058 [arXiv:1912.12546] [InSPIRE].

[59] W.D. Goldberger and I.Z. Rothstein, Dissipative effects in the worldline approach to black hole dynamics, Phys. Rev. D 73 (2006) 104030 [hep-th/0511133] [INSPIRE].

[60] R.A. Porto, Absorption effects due to spin in the worldline approach to black hole dynamics, Phys. Rev. D 77 (2008) 064026 [arXiv:0710.5150] [InSPIRE].

[61] W.D. Goldberger, J. Li and I.Z. Rothstein, Non-conservative effects on Spinning Black Holes from World-Line Effective Field Theory, arXiv:2012.14869 [INSPIRE].

[62] L. Blanchet, T. Damour and G. Esposito-Farese, Dimensional regularization of the third postNewtonian dynamics of point particles in harmonic coordinates, Phys. Rev. D 69 (2004) 124007 [gr-qc/0311052] [INSPIRE].

[63] S. Foffa and R. Sturani, Effective field theory calculation of conservative binary dynamics at third post-Newtonian order, Phys. Rev. D 84 (2011) 044031 [arXiv:1104.1122] [INSPIRE].

[64] S. Foffa and R. Sturani, Dynamics of the gravitational two-body problem at fourth post-Newtonian order and at quadratic order in the Newton constant, Phys. Rev. D 87 (2013) 064011 [arXiv:1206.7087] [INSPIRE]. 
[65] C.R. Galley, A.K. Leibovich, R.A. Porto and A. Ross, Tail effect in gravitational radiation reaction: Time nonlocality and renormalization group evolution, Phys. Rev. D 93 (2016) 124010 [arXiv: 1511.07379] [INSPIRE].

[66] S. Foffa, P. Mastrolia, R. Sturani and C. Sturm, Effective field theory approach to the gravitational two-body dynamics, at fourth post-Newtonian order and quintic in the Newton constant, Phys. Rev. D 95 (2017) 104009 [arXiv:1612.00482] [INSPIRE].

[67] R.A. Porto and I.Z. Rothstein, Apparent ambiguities in the post-Newtonian expansion for binary systems, Phys. Rev. D 96 (2017) 024062 [arXiv:1703.06433] [INSPIRE].

[68] T. Damour, P. Jaranowski and G. Schäfer, Nonlocal-in-time action for the fourth post-Newtonian conservative dynamics of two-body systems, Phys. Rev. D 89 (2014) 064058 [arXiv: 1401.4548] [INSPIRE].

[69] T. Marchand, L. Bernard, L. Blanchet and G. Faye, Ambiguity-Free Completion of the Equations of Motion of Compact Binary Systems at the Fourth Post-Newtonian Order, Phys. Rev. D 97 (2018) 044023 [arXiv: 1707.09289] [InSPIRE].

[70] S. Foffa and R. Sturani, Conservative dynamics of binary systems to fourth Post-Newtonian order in the EFT approach I: Regularized Lagrangian, Phys. Rev. D 100 (2019) 024047 [arXiv: 1903.05113] [INSPIRE].

[71] S. Foffa, R.A. Porto, I. Rothstein and R. Sturani, Conservative dynamics of binary systems to fourth Post-Newtonian order in the EFT approach II: Renormalized Lagrangian, Phys. Rev. D 100 (2019) 024048 [arXiv: 1903.05118] [INSPIRE].

[72] S. Foffa, P. Mastrolia, R. Sturani, C. Sturm and W.J. Torres Bobadilla, Static two-body potential at fifth post-Newtonian order, Phys. Rev. Lett. 122 (2019) 241605 [arXiv: 1902.10571] [INSPIRE].

[73] J. Blümlein, A. Maier and P. Marquard, Five-Loop Static Contribution to the Gravitational Interaction Potential of Two Point Masses, Phys. Lett. B 800 (2020) 135100 [arXiv: 1902.11180] [INSPIRE].

[74] S. Foffa and R. Sturani, Hereditary terms at next-to-leading order in two-body gravitational dynamics, Phys. Rev. D 101 (2020) 064033 [Erratum ibid. 103 (2021) 089901] [arXiv: 1907. 02869] [INSPIRE].

[75] J. Blümlein, A. Maier, P. Marquard and G. Schäfer, The fifth-order post-Newtonian Hamiltonian dynamics of two-body systems from an effective field theory approach: potential contributions, Nucl. Phys. B 965 (2021) 115352 [arXiv:2010.13672] [INSPIRE].

[76] J. Blümlein, A. Maier, P. Marquard and G. Schäfer, Testing binary dynamics in gravity at the sixth post-Newtonian level, Phys. Lett. B 807 (2020) 135496 [arXiv:2003.07145] [INSPIRE].

[77] J. Blümlein, A. Maier, P. Marquard and G. Schäfer, The 6th post-Newtonian potential terms at $O\left(G_{N}^{4}\right)$, Phys. Lett. B 816 (2021) 136260 [arXiv:2101.08630] [InSPIRE].

[78] D. Bini, T. Damour and A. Geralico, Sixth post-Newtonian local-in-time dynamics of binary systems, Phys. Rev. D 102 (2020) 024061 [arXiv: 2004.05407] [INSPIRE].

[79] L. Blanchet, S. Foffa, F. Larrouturou and R. Sturani, Logarithmic tail contributions to the energy function of circular compact binaries, Phys. Rev. D 101 (2020) 084045 [arXiv: 1912.12359] [INSPIRE]. 
[80] G. Kälin and R.A. Porto, From Boundary Data to Bound States, JHEP 01 (2020) 072 [arXiv: 1910.03008] [INSPIRE].

[81] G. Kälin and R.A. Porto, From boundary data to bound states. Part II. Scattering angle to dynamical invariants (with twist), JHEP 02 (2020) 120 [arXiv: 1911.09130] [INSPIRE].

[82] G. Kälin and R.A. Porto, Post-Minkowskian Effective Field Theory for Conservative Binary Dynamics, JHEP 11 (2020) 106 [arXiv:2006.01184] [INSPIRE].

[83] G. Kälin, Z. Liu and R.A. Porto, Conservative Dynamics of Binary Systems to Third Post-Minkowskian Order from the Effective Field Theory Approach, Phys. Rev. Lett. 125 (2020) 261103 [arXiv: 2007. 04977] [INSPIRE].

[84] G. Kälin, Z. Liu and R.A. Porto, Conservative Tidal Effects in Compact Binary Systems to Next-to-Leading Post-Minkowskian Order, Phys. Rev. D 102 (2020) 124025 [arXiv: 2008.06047] [INSPIRE].

[85] L. Blanchet, Gravitational Radiation from Post-Newtonian Sources and Inspiralling Compact Binaries, Living Rev. Rel. 17 (2014) 2 [arXiv:1310.1528] [INSPIRE].

[86] K. Yee and M. Bander, Equations of motion for spinning particles in external electromagnetic and gravitational fields, Phys. Rev. D 48 (1993) 2797 [hep-th/9302117] [INSPIRE].

[87] J. Steinhoff, Spin gauge symmetry in the action principle for classical relativistic particles, arXiv: 1501.04951 [INSPIRE].

[88] A.J. Hanson and T. Regge, The Relativistic Spherical Top, Annals Phys. 87 (1974) 498 [INSPIRE].

[89] H. Elvang and Y.-t. Huang, Scattering Amplitudes in Gauge Theory and Gravity, Cambridge University Press, (2015).

[90] Z. Bern, J.J. Carrasco, M. Chiodaroli, H. Johansson and R. Roiban, The Duality Between Color and Kinematics and its Applications, arXiv:1909.01358 [INSPIRE].

[91] J.M. Henn, Lectures on differential equations for Feynman integrals, J. Phys. A 48 (2015) 153001 [arXiv: 1412.2296] [INSPIRE].

[92] D. Neill and I.Z. Rothstein, Classical Space-Times from the S Matrix, Nucl. Phys. B 877 (2013) 177 [arXiv: 1304.7263] [INSPIRE].

[93] C. Cheung, I.Z. Rothstein and M.P. Solon, From Scattering Amplitudes to Classical Potentials in the Post-Minkowskian Expansion, Phys. Rev. Lett. 121 (2018) 251101 [arXiv: 1808.02489] [INSPIRE].

[94] Z. Bern, C. Cheung, R. Roiban, C.-H. Shen, M.P. Solon and M. Zeng, Scattering Amplitudes and the Conservative Hamiltonian for Binary Systems at Third Post-Minkowskian Order, Phys. Rev. Lett. 122 (2019) 201603 [arXiv:1901.04424] [InSPIRE].

[95] Z. Bern, C. Cheung, R. Roiban, C.-H. Shen, M.P. Solon and M. Zeng, Black Hole Binary Dynamics from the Double Copy and Effective Theory, JHEP 10 (2019) 206 [arXiv:1908.01493] [INSPIRE].

[96] D.A. Kosower, B. Maybee and D. O'Connell, Amplitudes, Observables, and Classical Scattering, JHEP 02 (2019) 137 [arXiv:1811.10950] [INSPIRE].

[97] B. Maybee, D. O'Connell and J. Vines, Observables and amplitudes for spinning particles and black holes, JHEP 12 (2019) 156 [arXiv:1906.09260] [INSPIRE]. 
[98] C.R. Galley and R.A. Porto, Gravitational self-force in the ultra-relativistic limit: the "large-N" expansion, JHEP 11 (2013) 096 [arXiv:1302.4486] [INSPIRE].

[99] B.R. Holstein and A. Ross, Spin Effects in Long Range Gravitational Scattering, arXiv:0802.0716 [INSPIRE].

[100] N.E.J. Bjerrum-Bohr, J.F. Donoghue and P. Vanhove, On-shell Techniques and Universal Results in Quantum Gravity, JHEP 02 (2014) 111 [arXiv:1309.0804] [INSPIRE].

[101] V. Vaidya, Gravitational spin Hamiltonians from the S matrix, Phys. Rev. D 91 (2015) 024017 [arXiv: 1410.5348] [INSPIRE].

[102] A. Guevara, Holomorphic Classical Limit for Spin Effects in Gravitational and Electromagnetic Scattering, JHEP 04 (2019) 033 [arXiv:1706.02314] [INSPIRE].

[103] M.-Z. Chung, Y.-T. Huang, J.-W. Kim and S. Lee, The simplest massive S-matrix: from minimal coupling to Black Holes, JHEP 04 (2019) 156 [arXiv: 1812.08752] [INSPIRE].

[104] A. Guevara, A. Ochirov and J. Vines, Scattering of Spinning Black Holes from Exponentiated Soft Factors, JHEP 09 (2019) 056 [arXiv:1812.06895] [INSPIRE].

[105] A. Guevara, A. Ochirov and J. Vines, Black-hole scattering with general spin directions from minimal-coupling amplitudes, Phys. Rev. D 100 (2019) 104024 [arXiv:1906.10071] [INSPIRE].

[106] N.E.J. Bjerrum-Bohr, P.H. Damgaard, G. Festuccia, L. Planté and P. Vanhove, General Relativity from Scattering Amplitudes, Phys. Rev. Lett. 121 (2018) 171601 [arXiv: 1806 .04920] [INSPIRE].

[107] A. Cristofoli, N.E.J. Bjerrum-Bohr, P.H. Damgaard and P. Vanhove, Post-Minkowskian Hamiltonians in general relativity, Phys. Rev. D 100 (2019) 084040 [arXiv:1906.01579] [INSPIRE].

[108] S. Caron-Huot and Z. Zahraee, Integrability of Black Hole Orbits in Maximal Supergravity, JHEP 07 (2019) 179 [arXiv: 1810.04694] [INSPIRE].

[109] N. Arkani-Hamed, Y.-t. Huang and D. O'Connell, Kerr black holes as elementary particles, JHEP 01 (2020) 046 [arXiv: 1906.10100] [INSPIRE].

[110] N.E.J. Bjerrum-Bohr, A. Cristofoli and P.H. Damgaard, Post-Minkowskian Scattering Angle in Einstein Gravity, JHEP 08 (2020) 038 [arXiv: 1910.09366] [INSPIRE].

[111] M.-Z. Chung, Y.-T. Huang and J.-W. Kim, Classical potential for general spinning bodies, JHEP 09 (2020) 074 [arXiv: 1908.08463] [INSPIRE].

[112] Y.F. Bautista and A. Guevara, From Scattering Amplitudes to Classical Physics: Universality, Double Copy and Soft Theorems, arXiv:1903.12419 [INSPIRE].

[113] Y.F. Bautista and A. Guevara, On the Double Copy for Spinning Matter, arXiv: 1908.11349 [INSPIRE].

[114] A. Koemans Collado, P. Di Vecchia and R. Russo, Revisiting the second post-Minkowskian eikonal and the dynamics of binary black holes, Phys. Rev. D 100 (2019) 066028 [arXiv: 1904.02667] [INSPIRE].

[115] H. Johansson and A. Ochirov, Double copy for massive quantum particles with spin, JHEP 09 (2019) 040 [arXiv: 1906.12292] [INSPIRE].

[116] P.H. Damgaard, K. Haddad and A. Helset, Heavy Black Hole Effective Theory, JHEP 11 (2019) 070 [arXiv:1908.10308] [INSPIRE]. 
[117] R. Aoude, K. Haddad and A. Helset, On-shell heavy particle effective theories, JHEP 05 (2020) 051 [arXiv: 2001.09164] [INSPIRE].

[118] K. Haddad and A. Helset, Tidal effects in quantum field theory, JHEP 12 (2020) 024 [arXiv : 2008. 04920] [INSPIRE].

[119] R. Aoude, K. Haddad and A. Helset, Tidal effects for spinning particles, JHEP 03 (2021) 097 [arXiv : 2012.05256] [INSPIRE].

[120] A. Cristofoli, P.H. Damgaard, P. Di Vecchia and C. Heissenberg, Second-order Post-Minkowskian scattering in arbitrary dimensions, JHEP 07 (2020) 122 [arXiv: 2003.10274] [INSPIRE].

[121] M.-Z. Chung, Y.-t. Huang, J.-W. Kim and S. Lee, Complete Hamiltonian for spinning binary systems at first post-Minkowskian order, JHEP 05 (2020) 105 [arXiv:2003.06600] [INSPIRE].

[122] Z. Bern, A. Luna, R. Roiban, C.-H. Shen and M. Zeng, Spinning Black Hole Binary Dynamics, Scattering Amplitudes and Effective Field Theory, arXiv:2005.03071 [INSPIRE].

[123] Z. Bern, H. Ita, J. Parra-Martinez and M.S. Ruf, Universality in the classical limit of massless gravitational scattering, Phys. Rev. Lett. 125 (2020) 031601 [arXiv:2002.02459] [INSPIRE].

[124] P. Di Vecchia, A. Luna, S.G. Naculich, R. Russo, G. Veneziano and C.D. White, A tale of two exponentiations in $\mathcal{N}=8$ supergravity, Phys. Lett. B 798 (2019) 134927 [arXiv: 1908.05603] [INSPIRE].

[125] A. Antonelli, A. Buonanno, J. Steinhoff, M. van de Meent and J. Vines, Energetics of two-body Hamiltonians in post-Minkowskian gravity, Phys. Rev. D 99 (2019) 104004 [arXiv: 1901.07102] [INSPIRE].

[126] A. Brandhuber and G. Travaglini, On higher-derivative effects on the gravitational potential and particle bending, JHEP 01 (2020) 010 [arXiv:1905.05657] [INSPIRE].

[127] C. Cheung and M.P. Solon, Classical gravitational scattering at $\mathcal{O}\left(G^{3}\right)$ from Feynman diagrams, JHEP 06 (2020) 144 [arXiv:2003.08351] [INSPIRE].

[128] J. Parra-Martinez, M.S. Ruf and M. Zeng, Extremal black hole scattering at $\mathcal{O}\left(G^{3}\right)$ : graviton dominance, eikonal exponentiation, and differential equations, JHEP 11 (2020) 023 [arXiv: 2005. 04236] [INSPIRE].

[129] C. Cheung and M.P. Solon, Tidal Effects in the Post-Minkowskian Expansion, Phys. Rev. Lett. 125 (2020) 191601 [arXiv: 2006. 06665] [INSPIRE].

[130] M. Accettulli Huber, A. Brandhuber, S. De Angelis and G. Travaglini, Eikonal phase matrix, deflection angle and time delay in effective field theories of gravity, Phys. Rev. D 102 (2020) 046014 [arXiv: 2006. 02375] [INSPIRE].

[131] Z. Bern, J. Parra-Martinez, R. Roiban, E. Sawyer and C.-H. Shen, Leading Nonlinear Tidal Effects and Scattering Amplitudes, arXiv:2010.08559 [INSPIRE].

[132] C. Cheung, N. Shah and M.P. Solon, Mining the Geodesic Equation for Scattering Data, Phys. Rev. D 103 (2021) 024030 [arXiv:2010.08568] [INSPIRE].

[133] A. Guevara, B. Maybee, A. Ochirov, D. O'connell and J. Vines, A worldsheet for Kerr, JHEP 03 (2021) 201 [arXiv:2012.11570] [INSPIRE].

[134] Z. Bern et al., Scattering Amplitudes and Conservative Binary Dynamics at $\mathcal{O}\left(G^{4}\right)$, Phys. Rev. Lett. 126 (2021) 171601 [arXiv:2101.07254] [INSPIRE]. 
[135] J. Vines, Scattering of two spinning black holes in post-Minkowskian gravity, to all orders in spin, and effective-one-body mappings, Class. Quant. Grav. 35 (2018) 084002 [arXiv: 1709.06016] [INSPIRE].

[136] J. Vines, J. Steinhoff and A. Buonanno, Spinning-black-hole scattering and the test-black-hole limit at second post-Minkowskian order, Phys. Rev. D 99 (2019) 064054 [arXiv: 1812.00956] [INSPIRE].

[137] E. Herrmann, J. Parra-Martinez, M.S. Ruf and M. Zeng, Gravitational Bremsstrahlung from Reverse Unitarity, Phys. Rev. Lett. 126 (2021) 201602 [arXiv:2101.07255] [InSPIRE].

[138] P. Di Vecchia, C. Heissenberg, R. Russo and G. Veneziano, Radiation Reaction from Soft Theorems, arXiv:2101.05772 [INSPIRE].

[139] G. Mogull, J. Plefka and J. Steinhoff, Classical black hole scattering from a worldline quantum field theory, JHEP 02 (2021) 048 [arXiv: 2010.02865] [INSPIRE].

[140] G.U. Jakobsen, G. Mogull, J. Plefka and J. Steinhoff, Classical Gravitational Bremsstrahlung from a Worldline Quantum Field Theory, Phys. Rev. Lett. 126 (2021) 201103 [arXiv:2101.12688] [INSPIRE].

[141] S. Mougiakakos, M.M. Riva and F. Vernizzi, Gravitational Bremsstrahlung in the Post-Minkowskian Effective Field Theory, arXiv:2102.08339 [INSPIRE].

[142] Y. Iwasaki, Quantum theory of gravitation vs. classical theory. Fourth-order potential, Prog. Theor. Phys. 46 (1971) 1587 [INSPIRE].

[143] O.B. Firsov, Determination of the forces acting between atoms using the differential effective cross-section for elastic scattering, Zh.Eksp.Teor.Fiz. 24 (1953) 279.

[144] D. Bini, T. Damour and A. Geralico, Sixth post-Newtonian nonlocal-in-time dynamics of binary systems, Phys. Rev. D 102 (2020) 084047 [arXiv:2007.11239] [INSPIRE].

[145] M. Tessmer, J. Hartung and G. Schäfer, Aligned Spins: Orbital Elements, Decaying Orbits, and Last Stable Circular Orbit to high post-Newtonian Orders, Class. Quant. Grav. 30 (2013) 015007 [arXiv:1207.6961] [INSPIRE].

[146] A. Le Tiec, L. Blanchet and B.F. Whiting, The First Law of Binary Black Hole Mechanics in General Relativity and Post-Newtonian Theory, Phys. Rev. D 85 (2012) 064039 [arXiv:1111.5378] [INSPIRE].

[147] E.T. Newman and A.I. Janis, Note on the Kerr spinning particle metric, J. Math. Phys. 6 (1965) 915 [INSPIRE].

[148] H.S. Chia, Tidal Deformation and Dissipation of Rotating Black Holes, arXiv: 2010.07300 [INSPIRE].

[149] A. Le Tiec, M. Casals and E. Franzin, Tidal Love Numbers of Kerr Black Holes, Phys. Rev. D 103 (2021) 084021 [arXiv: 2010.15795] [INSPIRE].

[150] P. Charalambous, S. Dubovsky and M.M. Ivanov, On the Vanishing of Love Numbers for Kerr Black Holes, JHEP 05 (2021) 038 [arXiv:2102.08917] [INSPIRE].

[151] T. Binnington and E. Poisson, Relativistic theory of tidal Love numbers, Phys. Rev. D 80 (2009) 084018 [arXiv:0906.1366] [INSPIRE].

[152] T. Damour and A. Nagar, Relativistic tidal properties of neutron stars, Phys. Rev. D 80 (2009) 084035 [arXiv:0906.0096] [INSPIRE]. 
[153] L. Hui, A. Joyce, R. Penco, L. Santoni and A.R. Solomon, Static response and Love numbers of Schwarzschild black holes, JCAP 04 (2021) 052 [arXiv: 2010. 00593] [INSPIRE].

[154] F.A. Berezin and M.S. Marinov, Particle Spin Dynamics as the Grassmann Variant of Classical Mechanics, Annals Phys. 104 (1977) 336 [INSPIRE].

[155] L. Blanchet, A. Buonanno and A. Le Tiec, First law of mechanics for black hole binaries with spins, Phys. Rev. D 87 (2013) 024030 [arXiv:1211.1060] [INSPIRE].

[156] D. Kosmopoulos and A. Luna, Quadratic-in-Spin Hamiltonian at $\mathcal{O}\left(G^{2}\right)$ from Scattering Amplitudes, arXiv:2102.10137 [INSPIRE].

[157] G. Passarino and M.J.G. Veltman, One Loop Corrections for $e^{+} e^{-}$Annihilation Into $\mu^{+} \mu^{-}$ in the Weinberg Model, Nucl. Phys. B 160 (1979) 151 [InSPIRE].

[158] V.A. Smirnov, Analytic tools for Feynman integrals, vol. 250, Springer (2012), [DOI] [INSPIRE]. 